\title{
Slip and no-slip temperature boundary conditions at the interface of porous, plain media : convection
}

\author{
M. SAHRAOUI and M. KAVIANY \\ Department of Mechanical Engineering and Applied Mechanics, The University of Michigan, \\ Ann Arbor, MI 48109, U.S.A.
}

(Received 1 June 1992 and in final form 9 March 1993)

\begin{abstract}
Near the interface of porous plain media, convective heat transfer may be noticeably affected by the nonuniformity of the phase distributions. The boundary effects are modeled by using interfacial slip or no-slip temperature boundary conditions. The latter uses a variable transverse total diffusivity giving a continuous variation of the temperature near and across the interface. The former uses a constant transverse total diffusivity which requires a temperature slip cross the interface (in order to obtain accurate heat flux calculations). In this study these boundary conditions are examined by the direct simulation of the momentum and energy equations for a model porous medium made of two-dimensional periodic arrangements of cylinders. The slip coefficient is found to depend on the bulk Peclet number $P e_{j}$, the ratio of solid to fluid conductivity $k_{\mathrm{s}} / k_{\mathrm{r}}$, and the gap size $h$. For the no-slip boundary condition, the magnitude and the distribution of $D_{\perp}(y) / \alpha_{\mathrm{f}}$ also depend on $P e_{1}, k_{\mathrm{s}} / k_{\mathrm{f}}$, and $h$. For a solid bounding surface, and when $k_{\mathrm{s}} / k_{\mathrm{f}}>1$, the effective transverse conductivity $k_{\mathrm{e} \perp} / k_{\mathrm{r}}$ dominates over the hydrodynamic dispersion, and therefore, the accurate description of the variation of $k_{\mathrm{e} 1}(y) / k_{\mathrm{f}}$ becomes critical. For a fluid bounding medium, the results show that $D_{\perp}(y)$ is nonuniform on both sides of the interface. The nonuniformity of $D_{\perp}(y)$ in the fluid medium is due to the local two dimensionality of the flow. The total diffusivity tensor $\mathbf{D}$ in the bulk of a two-dimensional periodic structure is also examined. The effects of the Reynolds number, Prandtl number, particle shape, particle arrangement, and flow direction, on the bulk value of $\mathbf{D}$ are examined. It is found that for oblique flows, the ensemble-averaged longitudinal total diffusivity $D_{i} / \alpha_{f}$, over the tilt angle, approaches a $P e_{1}$ relation instead of a $P e_{1}^{2}$ relation expected for periodic structures.
\end{abstract}

\section{INTRODUCTION}

THE SIMULTANEOUS presence of pore-level temperature and velocity gradients and the application of the local volume averaging technique results in the inclusion of the pore-level convection contribution as an enhanced diffusion (or dispersion). This enhanced diffusion is characterized by the total thermal diffusivity tensor given by

$$
\mathbf{D}=\frac{\mathbf{K}_{\mathrm{c}}}{\left(\rho c_{\mathrm{p}}\right)_{\mathrm{f}}}+\varepsilon \mathbf{D}^{\mathrm{d}}
$$

where $\mathbf{K}_{\mathrm{e}}$ is the effective conductivity tensor and $\mathbf{D}^{\mathrm{d}}$ is the hydrodynamic dispersion tensor. The hydrodynamic dispersion is similar to the thermal eddy diffusivity in turbulence and is a direct result of the transport occurring at length scales smaller than the selected local representative elementary volume used in the averaging. As with the eddy diffusivity in plain media, the hydrodynamic dispersion in porous media is also anisotropic because of its dependence on the Darcean flow direction (flow anisotropy) and the presence of anisotropy in the solid phase distribution (structural anisotropy). Near the bounding surfaces of porous media, both the solid phase and the porelevel velocity distributions are different than those in the bulk, and therefore, further anisotropy and nonuniformity are found in $\mathbf{D}$. This nonuniformity is treated by using the no-slip or the slip boundary conditions. In the no-slip boundary condition a variable $D_{\perp}(y)$ is used to model the nonuniformity near the interface and gives a continuous temperature distribution. In the slip boundary condition, a uniform $D_{\perp}$ is used which requires a slip in the interfacial temperature for the accurate prediction of the heat flux across the interface. Below, we discuss these boundary conditions and the available results for the bulk (far from the interface) value of $\mathbf{D}$.

\subsection{No-slip boundary condition}

The energy equation for a unidirectional steadystate fluid flow parallel and heat flow perpendicular to the interface, is given by

$$
\frac{\mathrm{d}}{\mathrm{d} y}\left[D_{\perp}(y) \frac{\mathrm{d}\langle T\rangle_{\mathrm{v}}}{\mathrm{d} y}\right]=0 .
$$

In the porous medium and far from the interface, $D_{\perp}(y)$ becomes the bulk transverse dispersion coefficient. In the fluid hounding medium and far from the interface $D_{\perp}(y)$ is equal to $\alpha_{\mathrm{f}}$. For a solid bounding medium, the hydrodynamic part of $D_{\perp}(y)$, i.e. $D_{\perp}^{\text {d }}(y)$, vanishes near the boundary duc to the noslip velocity boundary condition and $D_{\perp}(y)$ is solely due to conduction. 


\section{NOMENCLATURE}

$a_{i} \quad i=1,2, \ldots$, constants

$A_{\text {ls }} \quad$ solid-fluid interfacial area [ $\left.\mathrm{m}^{2}\right]$

$\mathbf{b}_{\mathrm{f}}, \mathbf{b}_{\mathrm{s}}$ closure vector functions [m]

c) specific heat capacity [ $\left.\mathrm{J} \mathrm{kg}^{\cdot{ }^{\prime} \mathrm{K}} \mathrm{K} \cdot \mathrm{'}\right]$

d cylinder diameter [m]

D $^{\mathrm{d}}$ hydrodynamic dispersion tensor [ $\mathrm{m} \mathrm{s}^{\cdots \cdot 2}$ ]

D total diffusivity tensor [ $\left.\mathrm{ms}^{2}\right]$

$D_{1} \quad$ transverse total diffusivity [m s $\left.{ }^{2}\right]$

$D$ longitudinal total diffusivity [m s $\left.{ }^{2}\right]$

$h \quad$ width of plain medium [m]

K. effective conductivity tensor $\left[\mathrm{W} \mathrm{m}{ }^{1} \mathrm{~K} \quad\right.$ ']

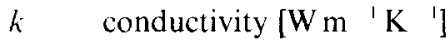

l cell dimension [m]

I. spatial periodicity vector [III]

$\mathbf{n}_{\mathrm{f}} \quad$ unit normal vector outward from fluid phase

$\mathbf{n}_{\mathrm{si}} \quad$ unit normal vector outward from solid phase

$P e_{1} \quad$ Peclet number, $u_{\mathrm{D}} l / x_{\mathrm{i}}$

$P e_{1} \quad$ Peclet number based on the flow along $x$-principal axis, $\langle u\rangle_{\mathrm{V}} l / \alpha_{1}$

p) $\quad$ pressure $\left[\mathrm{N} \mathrm{m}^{2}\right]$

$\mathrm{Pr} \quad$ Prandtl number, $v / \alpha_{\text {十 }}$

$r$ radial coordinate $[\mathrm{m}]$

$R \quad$ radius of circular cylinder $[\mathrm{m}]$

$R e_{1} \quad$ Reynolds number, $u_{0} l / v$

$T$ local temperature [K]

$T^{\prime} \quad$ deviation temperature [K]

$T_{\mathrm{h}} \quad$ temperature boundary condition at $y=h$

$\langle T\rangle_{\wedge}$, local area-averaged temperature in $x$-direction [K]

$\langle T\rangle_{\mathrm{V}}$ local volume-averaged temperature [K]

$\langle T\rangle_{\vee}$ local fluid-phase volume-averaged temperature. $\int_{i_{i}} T_{i} \mathrm{~d} V[\mathrm{~K}]$

u velocity vector [ $\left.\mathrm{ms}{ }^{\prime}\right]$

$\mathbf{u}^{\prime}$ deviation velocity vector, $\mathbf{u}-\langle\mathbf{u}\rangle\rangle_{\mathrm{v}}\left[\mathrm{m} \mathrm{s}^{-1}\right]$

$u \quad$ velocity in $x$-direction $\left[\mathrm{m} \mathrm{s}^{-1}\right]$ $u_{1}$ Darcean velocity [m s $\left.{ }^{1}\right]$

$i \quad$ velocity in $y$-direction $[\mathrm{ms}$ ']

$v$ local representative elementary volume $\left[\mathrm{m}^{3}\right]$

$V_{1} \quad$ local representative elementary fluid-phase volume [ $\left.\mathrm{m}^{3}\right]$

$V_{*} \quad$ local representative elementary solid-phase volume $\left[\mathrm{m}^{3}\right]$

$x, y \quad$ Cartesian coordinates $[\mathrm{m}]$.

Greek symbols

$x \quad$ thermal diffusivity $\left[\mathrm{m}^{2} \mathrm{~s} \cdot 1\right]$

$\alpha_{1} \quad$ temperature slip coefficient

$\Delta T \quad$ imposed temperature difference [K]

* porosity

$0 \quad$ tangential coordinate

$v \quad$ kinematic viscosity $\left[\mathrm{m}^{2} \mathrm{~s}\right]$

$\rho \quad$ densily $\left[\mathrm{kg} \mathrm{m}^{-3}\right]$.

Subscript

A Area

f fluid

max maximum

$s$ solid

V volume

$+\quad$ plain medium side

- porous medium side.

Superscripts

if fluid

s solid

deviation

$+\quad$ plain medium side

- porous medium side.

Others
\langle\rangle$^{\prime}$ fluid-phase volume averaged
\langle\rangle$_{A}$ area averaged
\langle\rangle$_{A}$, area averaged in $x$-direction
\langle\rangle$_{\mathrm{v}}$ volume averaged.

Several investigators have evaluated the distribution of $D_{\perp}(y)$ near the interface. Cheng and Vortmeyer [1] examine the nonuniformity of the hydrodynamic transverse dispersion coefficient near the bounding surface by using a variable local porosity along with the mixing length thcory of turbulence. A similar approach is used by Cheng and Hsu [2]. Tobis and Zilkowski [3] model the nonuniformity of $D_{\perp}^{\mathrm{d}}$ near the interface by using a nonuniform effective viscosity similar to the eddy viscosity in turbulence. By using the analogy between heat and momentum transfer they relate the viscosity to the hydrodynamic dispersion. Another approach is used by Hsu and Cheng [4] where they find expressions for the bulk $\mathbf{D}^{\downarrow}$ which depend explicitly on porosity. These expressions are used along with a variable porosity in order to model the nonuniformity of $D_{\mathrm{L}}^{\mathrm{d}}(y)$ and more details about the models mentioned above can be found in refs. $[5,6]$.

\subsection{Slip condition}

The difficulty with the prescription of the nonuniformity of $D_{1}(y)$ at the interface is bypassed by the extrapolation of the temperature away from the interface. The extrapolation of the temperature gives a temperature slip similar to the velocity slip suggested by Beavers and Joseph [7]. However, here the gradient in the porous medium side and far from the interface 
is used in order to determine the temperature slip. In this boundary condition, the temperature gradient in the porous medium and perpendicular to the interface is given by

$$
\left.\frac{\mathrm{d}\langle T\rangle_{\mathrm{v}}}{\mathrm{d} y}\right|_{r=0^{-}}=\frac{\alpha_{\mathrm{T}}}{\lambda}\left(T^{-}-T^{+}\right),
$$

where $\alpha_{\top}$ is the dimensionless slip coefficient, $T^{-}$is the porous medium interfacial temperature, $T^{+}$is the plain medium interfacial temperature, and $\lambda$ is a pore-level dimensionless length scale. Note that in convection, two boundary layers are formed around the porous plain interface, one on each side. Therefore, both $T^{+}$ and $T^{-}$are different than the actual interface temperature. For the bed of cylinders used in this study, we expect the length scale $\lambda$ to be the order of the unitcell length. Here it is taken to be unity (i.e. $\alpha_{T}$ is determined assuming a boundary-layer thickness equal to one unit cell size). This boundary condition is used along with the continuity of the heat flux, i.e.

$$
\left.D_{1} \frac{\mathrm{d}\langle T\rangle_{V}}{\mathrm{~d} y}\right|_{y=0^{-}}=\left.\alpha_{\mathrm{f}} \frac{\mathrm{d} T}{\mathrm{~d} y}\right|_{y=0^{-}} .
$$

Yagi and Kunii [8] performed experiments for an annular packed bed of spheres and used the slip boundary condition with a uniform $D_{\perp}(y)$ to find the heat flux at the boundary. Their results show that the slip coefficient behaves as a linear function of the particle Peclet number. Ofuchi and Kunii [9] used the same boundary condition but $D_{\perp}(y)$ was modeled by a step variation in order to account for the local porosity variation near the interface. Their step change is found by using a constant local porosity for the region near the boundary (which is different than the bulk value) and by using their empirical $D_{\mathrm{L}}=D_{\perp}(\varepsilon)$ relation.

\subsection{Bulk dispersion}

The determination of the bulk thermal diffusivity tensor has been the subject of many studies over the past five decades. The cross-sectional averaging of the molecular conduction-convection heat transfer for fully developed laminar flow in a tube by Taylor [10] results in the longitudinal (along the flow) hydrodynamic dispersion coefficient given by

$$
\frac{D_{\|}^{\mathrm{d}}}{x_{\mathrm{i}}}=\frac{P e_{\mathrm{d}}^{2}}{48}, \quad P e_{\mathrm{d}}=\frac{\langle u\rangle_{\mathrm{v}} d}{x_{\mathrm{f}}},
$$

where $\langle u\rangle_{\mathrm{V}}$ is the velocity. For turbulent flow, Taylor [11] uses an isotropic thermal eddy conductivity and he obtains a $P e$ relation. The $P e^{2}$ relation for laminar flows has also been found for ordered (simple cubic arrangement of spherical particles) porous media by Koch et al. [12]. As we will show, this is only true for ordered structures where the fluid particle path is not noticeably tortuous.

The theory of dispersion in periodic structures has been formulated by Brenner [13] by applying the method of moments. Koch et al. use this method to find closed-form solutions for the hydrodynamic dispersion tensor for orderly arranged beds of spheres and cylinders (two-dimensional). Their prediction of the longitudinal hydrodynamic dispersion coefficient gives a $P e^{2}$ relationship similar to that for tubes. Koch and Brady [14] also study the dispersion in random porous media using the ensemble averaging method and an asymptotic analysis for high porosities. They identify some of the physical mechanisms affecting dispersion. One of the mechanisms is the hold-up dispersion that occurs when a closed streamline region is present and the solute could only escape by molecular diffusion. This phenomenon has a $\mathrm{Pe}^{2}$ contribution to the longitudinal hydrodynamic dispersion. Another mechanism which has a $P e \ln (P e)$ contribution, results from the presence of a boundary layer on the particles. The mechanism is associated with the stochastic nature of the velocity field in random porous media and contributes with a simple Pe relation to $\mathbf{D}^{\mathbf{d}}$.

Carbonell and Whitaker $[15,16]$ derive the volume averaged energy equation using the volume averaging theory. Eidsath et al. [17] use this theory to evaluate D for a bed of non-conducting circular cylinders and their results nearly predict the trend shown by the experimental results of Gunn and Pryce [18]. They find that at high Peclet numbers $D / \alpha_{\mathrm{f}}$ follows a $P e^{1.7}$ relation. Edwards et al. [19] solve the same problem and examine the effects of porosity, particle arrangement, Prandtl number, and Reynolds number on the hydrodynamic dispersion tensor.

Here, direct numerical simulation of the flow and temperature fields and the application of the local volume-averaging technique are used to examine the effect of the pore-level flow regime (creeping and steady inertial regimes only) on the bulk $\mathbf{D}$. We also evaluate its nonuniformity and anisotropy near the bounding surfaces of an ordered porous medium (a two-dimensional porous medium made of cylindrical particles). In our previous communication the hydrodynamics [20] and conduction heat transfer [21], near the interface and in the bulk of such two-dimensional structures have been examined and reported.

\section{LOCAL VOLUME AVERAGING}

Here, the development of the volume-averaged energy equation in porous media is reviewed based on the development of Carbonell and Whitaker [16]. The hydrodynamic dispersion tensor is obtained in terms of the local variation of velocity and temperature. The local energy equation in the fluid and the solid is given by

$$
\frac{\partial T_{\mathrm{f}}}{\partial t}+\nabla \cdot \mathbf{u} T_{\mathrm{f}}=\nabla \cdot \alpha_{\mathrm{f}} \nabla T_{\mathrm{f}}
$$

and

$$
\frac{\partial T_{\mathrm{s}}}{\partial t}=\nabla \cdot \alpha_{\mathrm{s}} \nabla T_{\mathrm{s}} .
$$

The boundary conditions on $A_{\text {is }}$ 


$$
\mathbf{n}_{\mathrm{ts}} \cdot k_{\mathrm{f}} \nabla T_{\mathrm{f}}=\mathbf{n}_{\mathrm{s}} \cdot k_{\mathrm{s}} \nabla T_{\mathrm{s}} \text { and } T_{\mathrm{r}}=T_{\mathrm{s}}
$$

The volume averaged energy equation is obtained by averaging the local equations (6) and combining both of them using the assumption of the local thermal equilibrium. The obtained averaged equation is

$$
\begin{aligned}
& {\left[\varepsilon\left(\rho c_{p}\right)_{l}+(1-\varepsilon)\left(\rho c_{p}\right)_{N}\right] \stackrel{\partial\langle T\rangle_{Y}}{\partial t}+\left(\rho c_{p}\right)_{Y}\langle\mathbf{u}\rangle_{Y} \cdot \nabla\langle T\rangle_{Y}} \\
& =\nabla \cdot\left\{\left[k k_{\mathrm{r}}+(1-\varepsilon) k_{\mathrm{s}}\right] \nabla\langle T\rangle_{\nu}\right. \\
& \left.+\frac{k_{\mathrm{f}}-k_{\mathrm{s}}}{V} \int_{t_{\mathrm{s}}} \mathbf{n}_{\mathrm{ts}} T_{\mathrm{f}}^{\prime} \mathrm{d} A\right\}-\nabla \cdot\left\langle\mathbf{u}^{\prime} T_{\mathrm{f}}^{\prime}\right\rangle_{\mathrm{v}}
\end{aligned}
$$

where the volume average $\langle T\rangle_{Y}$ is given by

$$
\langle T\rangle_{v}=\frac{1}{V} \int_{V} T \mathrm{~d} V=(1-\delta)\langle T\rangle_{v}^{\mathrm{s}}+\varepsilon\langle T\rangle_{v}
$$

and $\langle\mathbf{u}\rangle_{\mathrm{Y}}$ is given by

$$
\langle\mathbf{u}\rangle_{v}=\frac{1}{V} \int_{l} \mathbf{u} \mathrm{d} V .
$$

and $T_{\mathrm{f}}^{\prime}$ and $\mathbf{u}^{\prime}$ represent the deviations about the intrinsic phase average and they are given by

$$
T_{i}^{\prime}=T-\langle T\rangle_{\mathrm{r}} \text { and } \quad \mathbf{u}_{\mathrm{f}}^{\prime}=\mathbf{u}-\langle\mathbf{u}\rangle_{\mathrm{v}}^{r}
$$

Note that $\langle T\rangle_{v}=\langle T\rangle_{v}^{\mathrm{r}}=\langle T\rangle_{\mathrm{v}}^{\mathrm{s}}$ using the local thermal equilibrium assumption. The last term in equation (8) represents the hydrodynamic dispersion caused by the interaction of the velocity and the temperature fields. A closure constitutive equation is introduced to relate the temperature deviation $T^{\prime}$ to the gradient of the averaged temperature using a first order approximation of $T$ about $\langle T\rangle_{v}$. The conditions are

$$
T_{\mathrm{f}}^{\prime}=\mathbf{b}_{\mathrm{f}} \cdot \nabla\langle T\rangle_{\mathrm{V}} \quad \text { and } \quad T_{\mathrm{s}}^{\prime}=\mathbf{b}_{\mathrm{s}} \cdot \nabla\langle T\rangle_{\mathrm{V}} .
$$

Then, the averaged energy equation (8) becomcs

$$
\begin{aligned}
{\left[\varepsilon+(1-\varepsilon) \frac{\left(\rho e_{\mathrm{p}}\right)_{s}}{\left(\rho c_{\mathrm{p}}\right)_{\mathrm{l}}}\right] \frac{\partial T\rangle_{\mathrm{v}}}{\partial t} } & +\langle\mathbf{u}\rangle_{\mathrm{v}} \cdot \nabla\langle T\rangle_{v} \\
& =\nabla \cdot\left(\mathbf{D} \cdot \nabla\langle T\rangle_{\mathrm{V}}\right),
\end{aligned}
$$

where $\mathbf{D}$ is the total diffusivity tensor given by cquation (1). Using the $\mathbf{b}$ vector, $\mathbf{K}_{\mathrm{c}}$ in equation (1) is given by

$$
\mathbf{K}_{\mathrm{e}}=\left[\varepsilon k_{\mathrm{f}}+(1-\varepsilon) k_{\mathrm{s}}\right] \mathbf{I}+\frac{k_{\mathrm{f}}-k_{\mathrm{s}}}{V} \int_{\mathrm{f}_{\mathrm{s}}} \mathbf{n}_{\mathrm{fs}} \mathbf{b}_{\mathrm{f}} \mathrm{d} \mathbf{A}
$$

and the hydrodynamic dispersion tensor $\mathbf{D}^{\mathrm{a}}$ in equation ( 1 ) is given by

$$
\mathbf{D}^{\mathrm{d}}=\frac{-1}{V_{\mathrm{r}}} \int_{l_{i}} \mathbf{u}^{\prime} \mathbf{b}_{\mathrm{i}} \mathrm{d} V .
$$

The $b$ vector in the solid and in the fluid is found by solving

$$
\mathbf{u}^{\prime}+\mathbf{u} \cdot \nabla \mathbf{b}_{i}=\alpha_{1} \nabla^{2} \mathbf{b}_{\mathrm{f}} \text { and } \nabla^{2} \mathbf{b}_{\mathrm{s}}=0
$$

The boundary conditions lor the $\mathbf{b}$ equations on $A$. are given by

$$
k_{\mathrm{f}} \mathbf{n}_{\mathrm{s}} \cdot \nabla \mathbf{b}_{\mathrm{f}}=k_{\mathrm{s}} \mathbf{n}_{f_{\mathrm{n}}} \cdot \nabla \mathbf{b}+\mathbf{n}_{\mathrm{fs}}\left(k_{\mathrm{w}}-k_{\mathrm{f}}\right)
$$

and

$$
\mathbf{b}_{1}=\mathbf{b}_{s} \text {. }
$$

At the boundaries of the unit cell the periodic boundary conditions are used.

\section{SOLUTION METHOD}

The total diffusivity tensor is evaluated for periodic arrangements of circular or square cylindrical particles. We solve the momentum, energy, and $\mathbf{b}$ equations for steady state, incompressible, and constant property flows. The equations are solved using the finite-difference approximations for a unit cell such as the one depicted in Fig. 1(a) using the finitevolume method developed by Patankar [22]. The circular cylinder inside the unit cell cannot be mapped accurately using a Cartesian grid. Therefore, we use a cylindrical grid around the cylinder which would map the surface of the cylinder exactly. Away from the cylinder surface and near the boundaries of the square cell we use a Cartesian grid. The momentum equation in the Cartesian and cylindrical coordinates and more details about the numerical method can be found in refs. $[5,20]$.

For the b equations, each component is solved for as a scalar. The equation for $b_{*}$ is given by

$$
u^{\prime}+u \frac{\partial b_{x}}{\partial x}+v \frac{\partial b_{x}}{\partial y}=\frac{1}{P_{e_{1}}}\left(\frac{\partial^{2} b_{x}}{\partial x^{2}}+\frac{\hat{\partial}^{2} b_{x}}{\partial y^{2}}\right) .
$$

The equation for $b_{y}$ is similar to that of $b_{x}$ except $u^{\prime}$ is replaced by $v^{\prime}$. The $\mathbf{b}$ vector is non-dimensionalized using $l$. For the interface problems. the energy equation is solved which is given by

$$
u_{\partial x}^{\partial T}+\partial T^{*}=\frac{1}{\partial y}\left(\begin{array}{l}
\hat{\partial}^{2} T \\
\hat{\partial} x^{2}
\end{array}+\frac{\partial^{2} T}{\partial y^{2}}\right) .
$$

The equation for $b_{x}$ in the cylindrical coordinates is given by

$$
\begin{aligned}
& u+u_{r} \frac{\partial b_{x}}{\partial x}+\frac{v_{y} b b_{x}}{r} \frac{\partial 0}{r} \\
& =\frac{1}{P e_{1}}\left[\begin{array}{ll}
1 & \partial \\
r & \partial r
\end{array}\left(\begin{array}{c}
\partial b_{x} \\
\partial r
\end{array}\right)+\frac{1}{\partial^{2}} \frac{\partial}{\partial \theta}\left(\begin{array}{c}
\partial b_{x} \\
\partial \theta
\end{array}\right)\right] .
\end{aligned}
$$

The energy equation in the cylindrical grid is given by

$$
u_{\mathrm{r}} \frac{\partial T}{\partial r}+\frac{v_{i}}{r} \frac{\partial T}{\partial \theta}=\frac{1}{P e_{1}}\left[\frac{1}{r} \frac{\partial}{\partial r}\left(r \frac{\partial T}{\partial r}\right)+\frac{1}{r^{2}} \frac{\partial}{\partial \theta}\left(\frac{\partial T}{\partial \theta}\right)\right] .
$$

Note that equations (20) and (21) are given for the fluid phase and they also apply for the solid phase where $u_{\mathrm{r}}=v_{i}=0$.

At the boundary of the cell the periodic boundary 
(a)

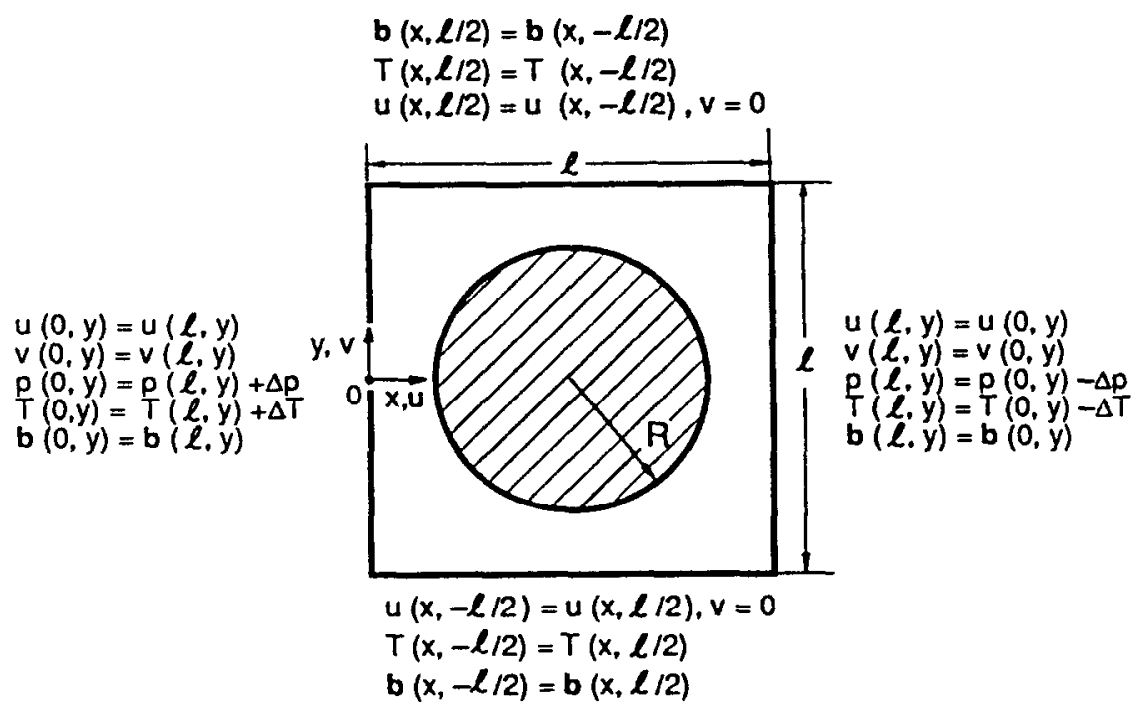

(b)
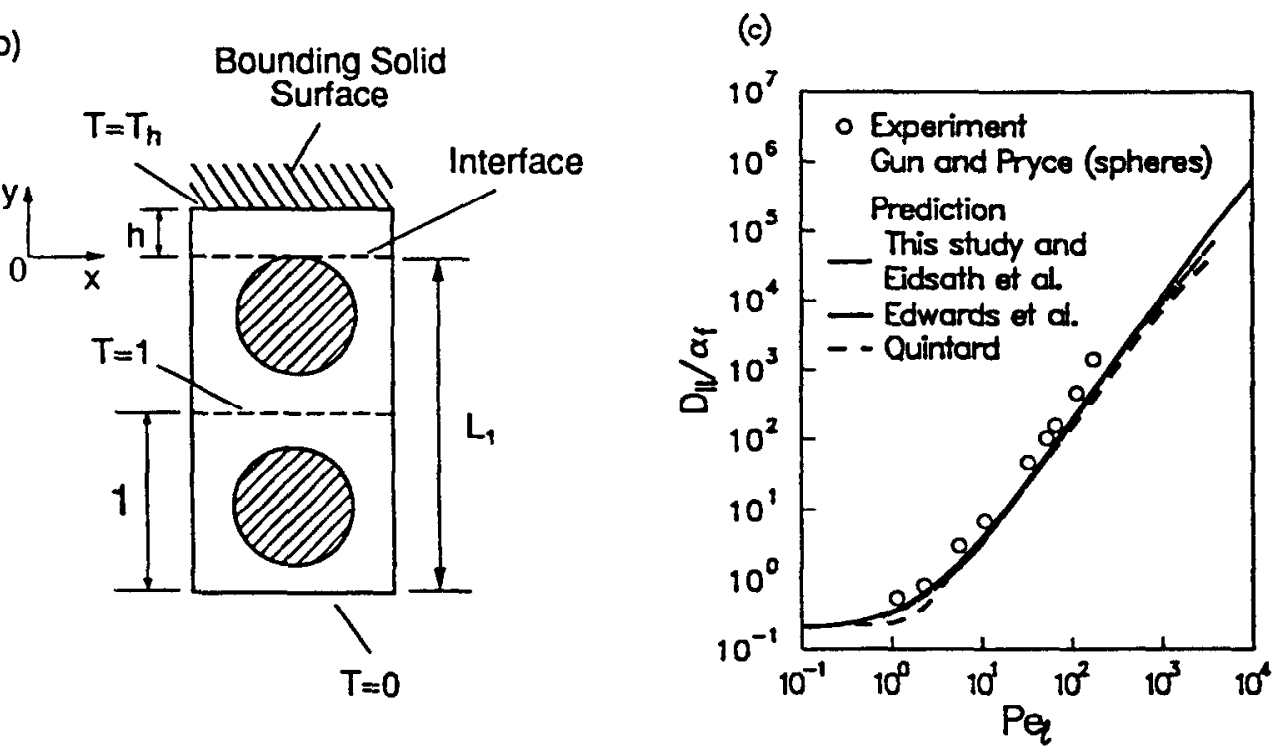

FIG. 1. (a) The unit cell and the periodic boundary conditions for the in-line arrangement of circular cylinders. (b) The boundary conditions for the interface model. (c) Comparison of the numerical results for $D / \alpha_{\mathrm{f}}$ of this study $\left(\varepsilon=0.38, R e_{1}=0.01\right.$, and $\left.k_{\mathrm{s}} / k_{\mathrm{f}}=0\right)$ and the numerical results of Edwards $e t a l$., Eidsath et al. and Quintard $e t$ al. and the experimental results of Gunn and Pryce.

conditions, as depicted in Fig. 1(a), are used for all the variables. When oblique flow is examined, the periodic boundary condition in the $y$-direction is similar to that applied in the $x$-direction in Fig. 1(a). The magnitude of flow in the $y$-direction is controlled by the pressure gradient imposed in that direction. At the surface of the cylinder, we use the no-slip condition for the velocity. For the $b_{x}$ and $b_{y}$, the boundary conditions are obtained from equation (17) and are given by

$$
\left.\frac{\partial b_{x}}{\partial r}\right|_{\mathrm{f}}=\left.\frac{k_{\mathrm{s}}}{k_{\mathrm{f}}} \frac{\partial b_{\mathrm{x}}}{\partial r}\right|_{\mathrm{s}}+\left(\frac{k_{\mathrm{s}}}{k_{\mathrm{f}}}-1\right) \cos (\theta)
$$

and

$$
\left.\frac{\partial b_{y}}{\partial r}\right|_{\mathrm{f}}=\left.\frac{k_{\mathrm{s}}}{k_{\mathrm{f}}} \frac{\partial b_{y}}{\partial r}\right|_{\mathrm{s}}+\left(\frac{k_{\mathrm{s}}}{k_{\mathrm{f}}}-1\right) \sin (\theta) .
$$

For the temperature equation, the harmonic mean is used and the boundary condition at the surface of the cylinder does not need any special treatment. When the energy equation is used to solve for the temperature distribution for the interface model, the boundary conditions given in Fig. 1(b) are used in the $y$-direction. In the $x$-direction, we use the periodic boundary condition given by 


$$
\Gamma(0,1)=T(1,1)
$$

The results are validated by comparing them to previous experimental and numerical results as shown in Fig. 1(c). The numerical results predict lower $D_{1} / \alpha_{f}$ than the experiments of Gunn and Pryec for the simple cubic packing of spherical particles $(z=0.48)$. This discrepancy is due to the difference in flow and temperature fields for the in-line arrangement of circular cylinders and that of simple cubic arrangement of spherical particles. When we compare our numerical results to those of Eidsath et al. we find very good agreement and in Fig. 1(c) we present the two results in terms of only one curve (present results) in order to reduce the complexity of the graph. Figure l(c) also shows that the present numerical results are in very good agreement with those of Quintard [23] and Edwards et $a$. for $P c_{1}<10^{3}$. For higher Peclet numbers, the deviation between the various numerical results increases because of the inaccuracies that are present in the numerical methods. Note that for the results of Edwards et al., we moditied their Peclet number definition in order to obtain matching results. They claim that their results are presented in terms of the fluid phase average velocity but we believe that they are presented in terms of the atverage velocity over both phases.

The solid phase nonuniformity near the porous plain media interface is examined using the model depicted in Fig. 1(b). The local solution for the temperature is found using equations (19) and (2l) with the boundary conditions given in Fig. 1(b). The slip and no-slip boundary conditions at the interface of porous plain media are examined after averaging is performed using a variable averaging volume. Away from the interface, where the solid phase distribution is uniform, an averaging volume having a unit-cell size is used. This averaging volume is defined by

$$
\langle T\rangle_{\vee}(x, y)=\int_{-0.5}^{0.5} \int_{-0.5}^{0.5} T\left(x+x^{\prime}, y^{\prime}+y^{\prime}\right) \mathrm{d} x^{\prime} \mathrm{d} y^{\prime}
$$

At the interface and in the plain medium, the averaging volume is zero and an area average of the local temperature is taken. The area-averaged temperature (averaged along the $x$-direction) is defined by

$$
\langle T\rangle_{\mathrm{A}_{3}}(x, y)=\int_{0.5}^{0.5} T\left(x+x^{\prime}, y\right) \mathrm{d} x^{\prime} .
$$

For the no-slip model a smoothly varying and well behaved averaged solution is expected. This is found by applying a variable averaging volume near the interface. This variable averaging volume allows the transition between the area average at the interface (i.e. zero averaging volume) to a single unit-cell size averaging volume one half cell size away from the interface. Therefore, the size of this averaging volume is taken as $-2 y$ for $-1 / 2 \leqslant y \leqslant 0$ and it becomes the unit-cell size for $1 \leqslant-1: 2$. The volume averaged temperature for $-1 / 2 \leqslant y \leqslant 0$ is given by

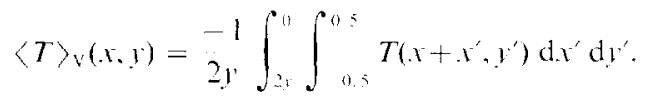

Further discussion about the use of the variable averaging can be found in ref. [20].

For conduction, the distributions of $b_{x}$ and $b_{y}$ within the unit cell are similar to that for $T^{\prime}$, and therefore, $\mathbf{b}$ or $T$ can he used to determine $\mathbf{K}_{\mathrm{c}}$. For high Peclet numbers, using the $T^{\prime}$ field becomes difficult because of the large temperature gradient near the flow exit boundary of the cell, and therefore, $b$ equations must be used. To demonstrate the distributions of $b_{x}$ and $b_{1}$, examples are given in Fig. 2 . The results are for various flow and bed parameters. In Fig. 2(a) the effect of the Peclet number on the distribution of $b_{\gamma}$ is shown for the in-line arrangement of cylinders and for $P_{\ell_{1}}=1$ and $10^{2}$. As the Peclet number increases both the distribution and the magnitude of $b_{x}$ change significantly. The effect of $k_{s} / k_{\mathrm{l}}$ is shown in Fig. 2(b) for $P e_{1}=1$ and $10^{2}$ and $k_{\mathrm{s}} / k_{\mathrm{f}}=10^{2}$. For $P e_{1}=1$, the effect of convection is negligible and the $b_{x}$ distribution is similar to that for conduction. For $P e_{1}=10^{2}$, the $b_{x}$ distribution changes significantly and becomes nearly similar to that of $k_{\mathrm{s}} / k_{\mathrm{f}}=1$ shown in Fig. 2(a). The effect of the particle arrangement on the distribution of $b_{x}$ is shown in Fig. 2(c). The results show a significant difference between the staggered and the inline arrangements of cylinders, especially at large Peclet numbers. As it will be shown below, this difference causes $D_{\|}^{d} / x_{f}$ for the two arrangements to be different by orders of magnitude. In the transverse direction, the $h_{1}$. field does not show a strong dependency on the Peclet number as shown in Fig. 2(d) for $P e_{1}=1$ and $10^{2}$.

\section{BULK DISPERSION TENSOR}

In this section, we examine $\mathbf{D}$ for the bulk porous medium and for the different structure and flow parameters. The structure parameters used are $k_{\mathrm{s}} / k_{\text {; }}$ and $\varepsilon$ and the flow parameters are $P e_{1}, R e_{1}$, and the flow direction. We first consider the longitudinal total diffusivity $D_{\uparrow}$ and the transverse total diffusivity $D$.

\subsection{Longitudinal component}

The total dispersion tensor, away from the interface, is examined for a bed of circular and square cylinders. There are two mechanisms that contribute to the hydrodynamic dispersion. The first mechanism is due to the velocity gradient in the pore caused by the no velocity slip occurring at the particle surface and also due to the tortuous fluid particle path caused by the solid particle arrangement. The second mechanism is due to the presence of a flow recirculation region, i.e. presence of closed streamlines. The heat transfer out of this region occurs only by molecular 
(a)
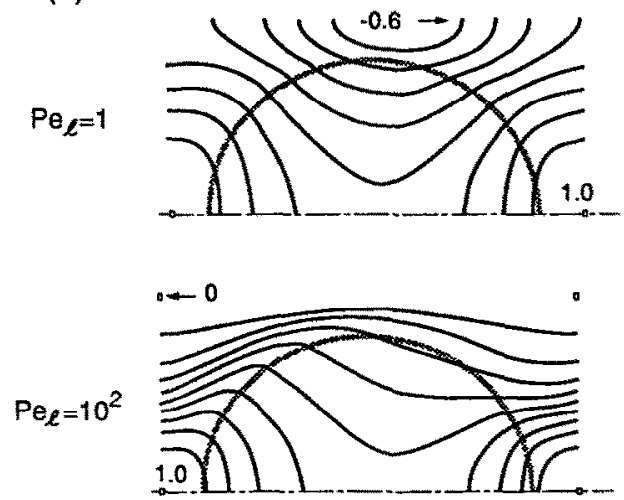

(c)

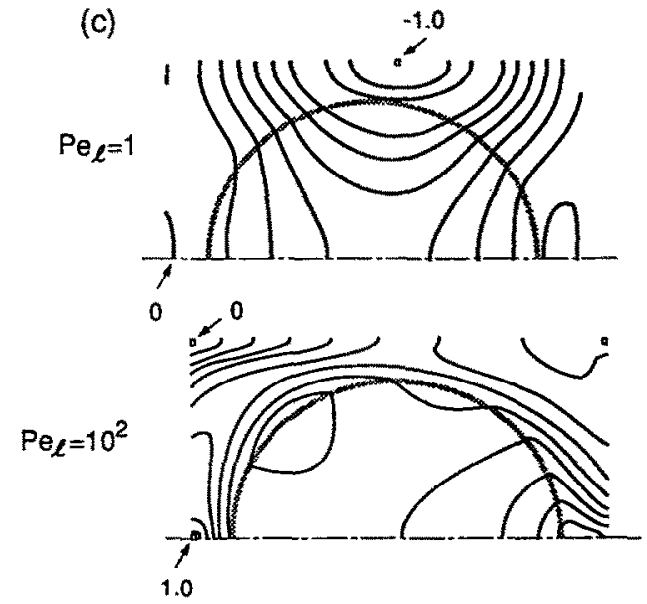

(b)
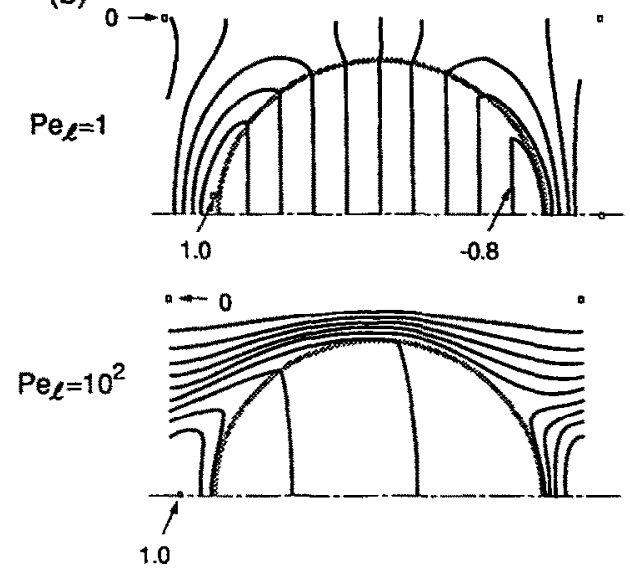

(d)
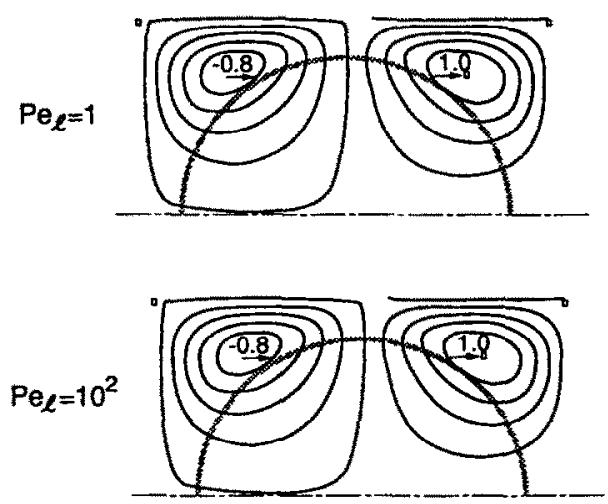

FIG. 2. (a) Contours of constant $b_{s} /\left|b_{x_{m a x}}\right|$ for the in-line arrangement of cylinders and for $P e_{1}=1$ and $10^{2}$ $\left(\varepsilon=0.5, k_{\mathrm{s}} / k_{\mathrm{f}}=1\right.$, and $\left.R e_{1}=0.01\right)$. (b) Effect of $k_{s} / k_{\mathrm{f}}$ on $b_{s} /\left|b_{\mathrm{x}_{\max }}\right|$ for the in-line arrangement of cylinders, with $k_{s} / k_{r}=100$ and for $P e_{1}=1$ and $10^{2}\left(\varepsilon=0.5\right.$ and $\left.R e_{1}=0.01\right)$. (c) Effect of Peclet number on $h_{s} /$ $\left|b_{x_{\text {mit }}}\right|$ for the staggered arrangement of cylinders and for $P e_{1}=1$ and $10^{2}\left(\varepsilon=0.5, k_{v} / k_{\mathrm{f}}=1\right.$, and $\left.R e_{1}=0.01\right)$. (d) Contours of constant $b_{1} / \mid b_{i}$, for the in-line arrangement of cylinders and for $P e_{1}=1$, and $10^{2}(\varepsilon=0.5$. $k_{\mathrm{s}} / k_{\mathrm{f}}=1$, and $R e_{1}=0.01$ ).

diffusion. This dispersion mechanism is especially important for some periodic structures, where vortices can exist between adjacent cylinders.

To compare these different mechanisms, we have computed the recirculation region contribution and that from the remaining region separately for an arrangement of square cylindrical particles. The results show that for $\varepsilon=0.5$ and $P e_{1}=10^{3}$, the contribution of the recirculation region is about $90 \%$ of the total.

4.1.1. Porosity. Since the recirculation region covers a larger portion of the pore volume and the velocity gradients become more pronounced as the spacing between the particles decreases, $D^{d}$ is expected to increase with a decrease in porosity. The increase in $D^{\mathrm{d}} / \alpha_{\mathrm{r}}$ with decrease in porosity, for in-line arrangement of cylinders, is shown in Fig. 3(a) and further results for $D^{\mathrm{d}} / \alpha_{r}$ are given in ref. [24]. Note that the variation in $D_{i l} / \alpha_{i}=1+\delta D^{\mathrm{d}} / \alpha_{\mathrm{i}}$ with respect to $P e_{1}$ is shown in Fig. 3(a). These results show that the high $P e_{1}$ asymptotic behavior is independent of the porosity, i.e. the results for all porosities show that $D_{i} \alpha_{f}$ is proportional to $P e_{1}^{u}$, for high $P e_{1}$. The results depicted in Fig. 3(a) also show that the change in $D_{\mathbb{l}} / \alpha_{\mathrm{f}}$, caused by the porosity variation, is not very significant compared to the variation with respect to $P e_{1}$. For example, the difference in $D_{4}^{d} / \alpha_{r}$ between $\varepsilon=0.5$ and 0.95 is only about $60 \%$. As will be discussed below, the particle arrangement or the flow direction can change $D_{\|}^{\mathrm{d}} / \alpha_{\mathrm{f}}$ by orders of magnitude.

4.1.2. Reynolds and Prandtl numbers. So far, the Peclet number $P e_{1}=R e_{1} P r$ is varied by changing the Prandtl number while keeping the Reynolds number the same. In order to evaluate the effect of the flow inertia on $D_{\mid} / \alpha_{f}$, we now vary the Reynolds number while keeping the Prandtl number the same. The 
(a)

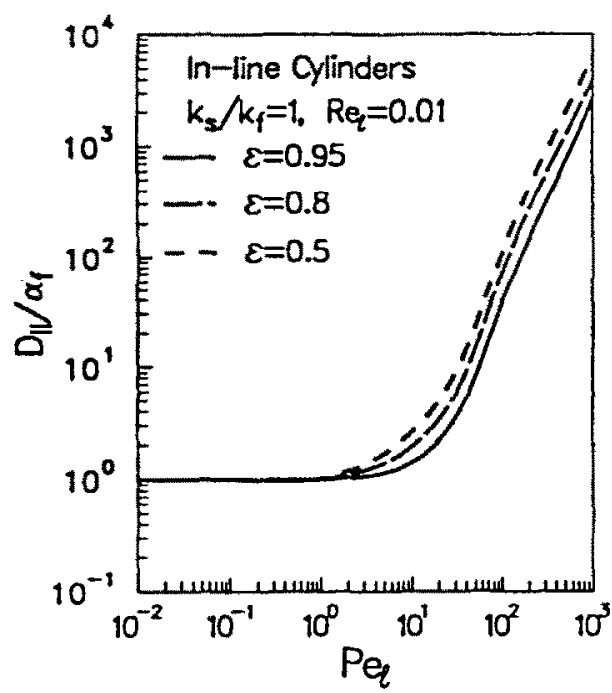

(b)

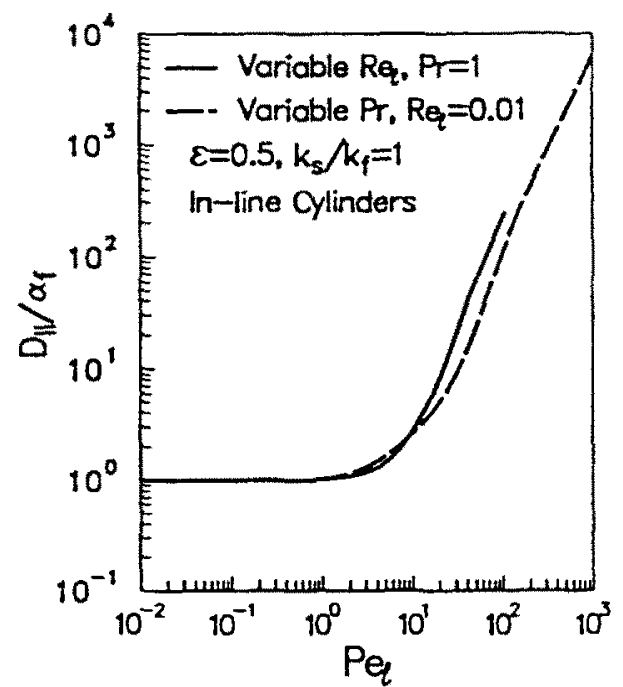

(c)

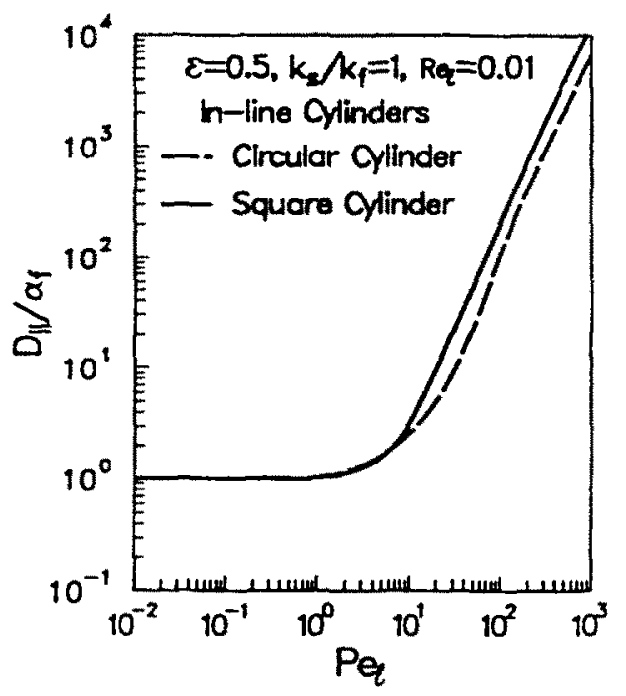

FiG. 3. (a) Effect of the porosity on variation of $D_{1} / \alpha_{i}$ for in-line arrangement of circular cylinders. (b) Variation of $D / \alpha_{f}$ as a function of the Peclet number for constant $P r$ and constant $R e_{1}$ for in-line arrangement of circular cylinders. (c) Comparison of the variation of $D_{\|} / \alpha_{f}$ with respect to $P e_{1}$ for in-line arrangement of square and circular cylinders.

results are shown in Fig. 3(b) for $P e_{1} \leqslant 10^{2}$. Note that the flow becomes unsteady for $R e_{1} \geqslant 150$, as discussed in ref. [20]. Also shown in Fig. 3(b) are the results of varying $\operatorname{Pr}$ while keeping $R e_{1}$ the same. The results show that at high Peclet numbers the power $a$ in the $P e_{3}^{e q}$ relation is the same for both variable $P r$ and $R e_{1}$. However, due to the inertial effects, varying the Reynolds number results in a higher $D_{1} / \alpha_{f}$. The inertial effects on the flow field for in-line arrangement of circular cylinders are discussed in ref. [20]. For low Reynolds numbers, separation occurs away from the tip of the cylinder and the streamlines curve around the cylinder. For higher Reynolds number, the flow separates before the tip of the cylinder making the flow field nearly rectilinear. This earlier flow separation causes an increase in the extent of the recirculation region and as the Reynolds number increases, $D_{i}^{d} / \alpha_{i}$ increases.

4.1.3. Particle shape. The effect of the particle shape on $D^{\mathrm{d}} / \alpha_{\mathrm{f}}$ is examined using circular and square cylinders. The results for square and circular cylinders are compared in Fig. 3(c). The results show that due to a larger recirculation region between the cylinders, $D^{\mathrm{d}} / \alpha_{\mathrm{r}}$ for square cylinders, and at high $P e_{i}$, is larger. For the square cylinders, the flow is partly rectilinear and can be compared to the flow between two parallel 
plates. However, for the square cylinders $D_{\|}^{d} / \alpha_{f}$ is greatly affected by the recirculation, while for a straight channel the dispersion is only caused by the velocity gradient in the channel. A channel having the same size as the gap between the square cylinders will have a $D_{\|}^{\mathrm{d}} / \alpha_{\mathrm{f}}$ which is lower by about $80 \%$.

The Reynolds number effect is also studied for the square cylinders, but the flow field does not change significantly with the Reynolds number (due to the absence of a curvature on the surface). Then, the results for $D_{\|}^{\mathrm{d}} / \alpha_{\mathrm{f}}$ are nearly the same for the variable Reynolds and Prandtl number.

4.1.4. Particle conductivity. The effect of solid to fluid conductivity ratio $k_{\mathrm{s}} / k_{\mathrm{f}}$ on $D_{\|} / \alpha_{\mathrm{f}}$ has been recently studied by Yuan et al. [25]. In their model, a thick wall capillary tube is used in order to evaluate this effect. They obtain the same $P e_{1}$ dependency as that of Taylor (equation (5)). Moreover, they find that at high Peclet numbers, $D_{\|} / \alpha_{\mathrm{f}}$ decreases with increasing $k_{\mathrm{s}} / k_{\mathrm{f}}$ while at low Peclet numbers it increases. Our results show the same trend for the inline arrangement of circular cylinders. The variations of $D_{\|} / \alpha_{\mathrm{f}}$ with respect to $k_{\mathrm{s}} / k_{\mathrm{f}}$ for $P e_{1}=1$ and $10^{3}$ are shown in Figs. 4(a) and (b). For low Peclet number flows, i.e. $P e_{1}<10, D_{\|} / \alpha_{f}$ increases as shown in Fig. 4(a). This is expected because for low Peclet numbers, the hydrodynamic effects are not very significant and the transport is diffusion controlled. In this regime, as shown in the discussion of conduction heat transfer in ref. [21], the effective conductivity initially increases with an increase in $k_{\mathrm{s}} k_{\mathrm{f}}$ and then reaches an asymptote. As the Peclet number increases, convection dominates and the effect of $k_{\mathrm{s}} / k_{\mathrm{f}}$ on $D_{\mathrm{i}} / \alpha_{\mathrm{f}}$ is noticeably different. The transition between the high and low Peclet number regimes occurs around $P e_{1}=10$ as shown in ref. [24]. For higher Peclet numbers $\left(P e_{1}>10\right), D_{i}^{\mathrm{d}} / \alpha_{\mathrm{f}}$ is enhanced by lowering $k_{\mathrm{s}} / k_{\mathrm{f}}$, as shown in Fig. 4(b), for $P e_{1}=10^{3}$ which is consistent with the results of Yuan et al.

4.1.5. Particle arrangement. As the pore geometry changes, so does the flow field, and therefore, the dispersion tensor changes. Here, we study the effect of the particle arrangement on $D_{\|} / \alpha_{\mathrm{f}}$ by examining the in-line and the staggered arrangements of particles. In the staggered arrangement, two adjacent columns of in-line cylinders are shifted with respect to each other by a distance of half a cell size. The results for $D_{\mathrm{i}}^{\mathrm{d}} / \alpha_{\mathrm{f}}$ are shown in Fig. 4(c). At $P e_{1}=10^{3}$ and for the staggered arrangement, $D_{\|}^{\mathrm{d}} / \alpha_{\mathrm{r}}$ is lower by about two orders of magnitude for the same values of $\varepsilon, k_{\mathrm{s}} / k_{\mathrm{f}}$, and $R e_{1}$. This difference is attributed to two effects. First, for the staggered arrangement the recirculation region, that is present between the two adjacent particles for the in-line arrangement, is not present (as shown in ref. [20]) resulting in a lower $D_{\|} / \alpha_{\mathrm{f}}$. The second effect, which is more significant, is due to the interruptions made to the motion of fluid particles by the staggered solid particles. For the in-line arrangement, the velocity distribution does not change significantly along the flow direction. For the staggered arrangement, the fluid particles follow a tortuous path and undergo periodic and substantial change in direction and magnitude of their velocity. This results in a lower value for $D_{\|} / \alpha_{f}$ compared with the in-line arrangement. The substantial change in the velocity of the fluid particles occurs also in the disordered porous media. From the results shown in Fig. 4(c) for high $P e_{1}$, the exponent $a_{2}$ in $D_{1} / \alpha_{\mathrm{f}}=a_{1} P e_{1}^{a_{2}}$ is 1.26 for $\varepsilon=0.5$. This trend is also found in the experimental results of Gunn and Pryce [18] for the rhombohedral arrangement of spherical particles. In the existing literature these experimental results have not been compared to any predictions. This is because a $P e_{1}^{2}$ relation had been expected for all periodic arrangements of particles. We show that a $P e_{1}^{2}$ relation is not found for any periodic structure. Table 1 gives the coefficients $a_{1}$ and $a_{2}$ in $D_{\|} / \alpha_{1} P e_{1}^{a_{2}}$, for different $\varepsilon$ and for the staggered and the in-line arrangements of cylinders. The results show that as $\varepsilon$ increases $a_{2}$ increases and approaches a value of 2 , as expected for a periodic structure. Note that, $D_{\|} / \alpha_{\mathrm{f}}$ is significantly lower for the staggered than the in-line arrangement.

4.1.6. Flow direction. So far, $D_{\|} / \alpha_{f}$ has only been examined for Darcean flows along the principal axes of the solid matrix (or bed). The off principal axes flows have been examined by Koch et al., for the inline arrangement of circular cylinders. Their results show that with a slight deviation from the principal axes, instead of a monotonic increase of $D^{\mathrm{d}} / \alpha_{\mathrm{f}}$ with respect to $P e_{1}$, an asymptote is reached for high Peclet numbers (c.g. for $P e_{1} \geqslant 10^{3}$ and a tilt angle of $0.2^{\circ}$ ). This trend is not found here, as shown in Fig. 4(d), where we present the variation of $D_{\|}^{\mathrm{d}} / \alpha_{\mathrm{f}}$ with respect to $P e_{\left.\right|_{x}}$ (i.e. the Peclet number based on the flow along $x$-principal axis) for the in-line arrangement of cylinders. The results are presented for several tilt angles from $0^{\circ}$ up to $89.4^{\circ}$. At high values of $P e_{1}$, as the tilt angle increases, $D_{\|} / \alpha_{\mathrm{f}}$ decreases. This is because the $y$-direction flow eliminates the recirculation region between the cylinders and creates more tortuous fluid particle paths. Note that a tilt in Darcean flow corresponds to staggering of particles. Figure $4(\mathrm{~d})$ also shows that at high Peclet numbers, $D_{1} / \alpha_{f}$ approaches an asymptotic behavior of the form $D / \alpha_{f}=a_{1} P e_{1}^{a_{2}}$. The coefficients $a_{1}$ and $a_{2}$ are computed for different tilt angles and are shown in Table 2 . These results show that the exponent $a_{2}$ decreases drastically for small tilt angles and becomes nearly unity for $\langle v\rangle_{\mathrm{V}} /\langle u\rangle_{\mathrm{V}}=0.05$ to 0.1 . As the tilt angle further increases, the exponent first increases, also seen in Fig. 4(d), and then decreases again. From Table 2, we find that for $\langle v\rangle_{\mathrm{v}} /\langle u\rangle_{\mathrm{v}}=0.9$ and $1, D_{\|} / \alpha_{\mathrm{f}}$ is larger compared to the results for 0.7 . This is because as the tilt angle changes the tortuosity and the magnitude of the velocity gradient change to enhance $D_{y}^{\mathrm{d}} / \alpha_{\mathrm{f}}$. For $\langle v\rangle_{\mathrm{v}} /\langle u\rangle_{\mathrm{v}}=10^{2}, \quad D_{: \mid} / \alpha_{\mathrm{f}}$ is larger compared to $\langle v\rangle_{\mathrm{v}} /\langle u\rangle_{\mathrm{v}}=10$. This is because the overall Peclet number, based on the total velocity, is about 10 times larger compared to that for $\langle v\rangle_{\mathrm{V}} /\langle u\rangle_{\mathrm{V}}=10$, while the tilt angles are not very different. 
(a)

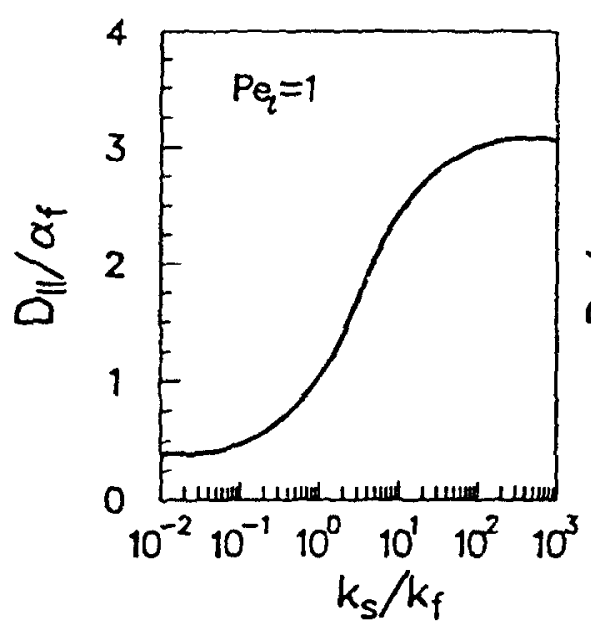

(c)

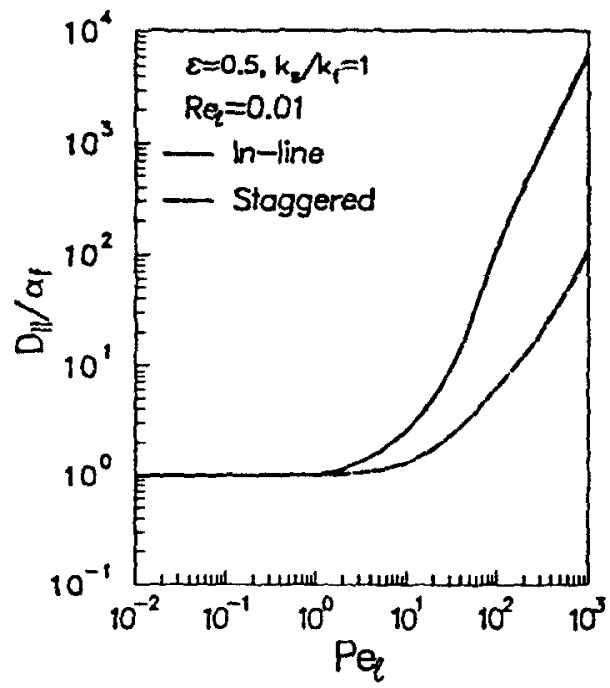

(b)

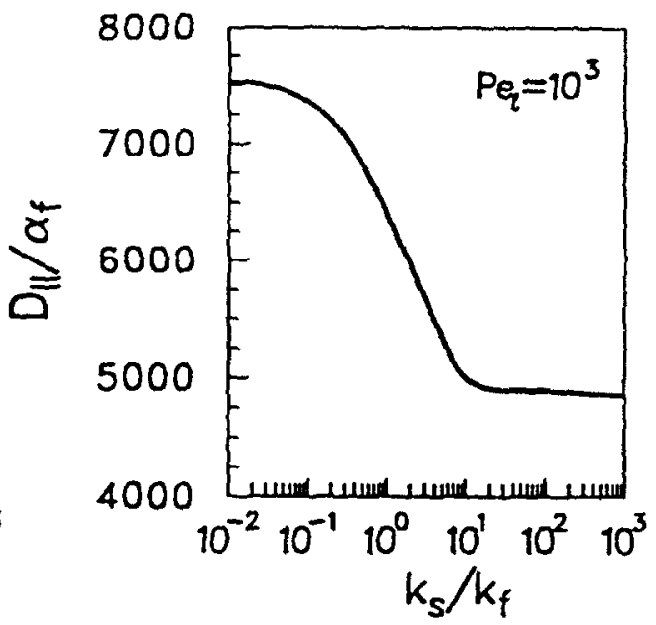

(d)

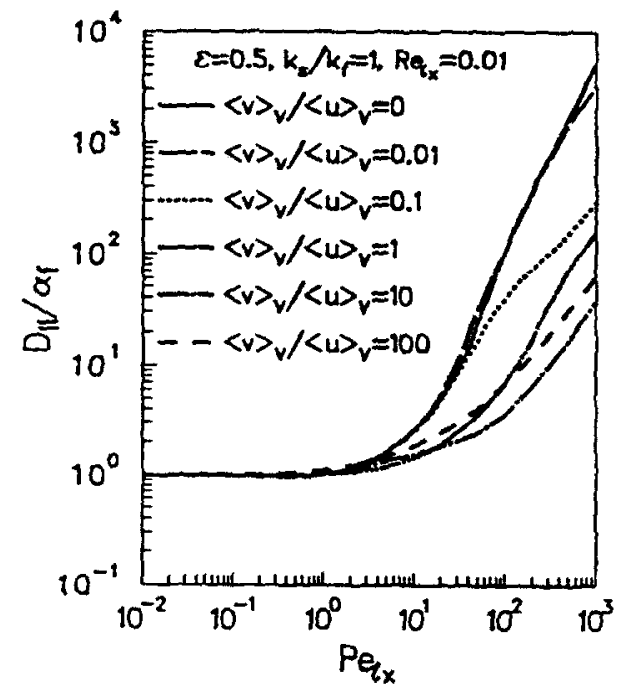

F1G. 4. (a), (b) Effect of $k_{s} / k_{f}$ on $D / \alpha_{f}$ for in-line arrangement of circular cylinders $\left(\varepsilon=0.5\right.$ and $R e_{1}=0.01$ ) for $P e_{1}=1$ and $P e_{1}=10^{3}$, respectively. (c) Effect of the particle arrangement on $D_{\|} / \alpha_{i}$ for variable $P e_{1}$ using in-line and staggered arrangements of circular cylinders. (d) Effect of the flow tilt on $D: \alpha_{f}$ for inline arrangement of circular cylinders.

Table 1. Effect of particle arrangement on the coefficients in $D_{\|} / \alpha_{f}=a_{1} P e_{1}^{a_{2}}$, for $k_{\mathrm{s}} / k_{f}=1.0$ and $R e_{1}=0.01$. The results are for $10^{2} \leqslant P e_{1} \leqslant 10^{3}$

\begin{tabular}{ccccc} 
& \multicolumn{2}{c}{ In-line } & \multicolumn{2}{c}{ Staggered } \\
\cline { 2 - 4 } & $a_{2}$ & $a_{1}$ & $a_{2}$ & $a_{1}$ \\
\hline 0.5 & 1.71 & 0.048 & 1.26 & 0.018 \\
0.6 & 1.68 & 0.049 & 1.37 & 0.013 \\
0.7 & 1.68 & 0.044 & 1.43 & 0.011 \\
0.8 & 1.67 & 0.039 & 1.49 & 0.0080 \\
0.95 & 1.86 & 0.0076 & 1.54 & 0.0042 \\
\hline
\end{tabular}

Table 2. Effect of flow direction on the coefficients in $D_{\mathrm{i}} / \alpha_{\mathrm{f}}=a_{1} P e_{\mathrm{i}}^{a_{2}}$, for $k_{\mathrm{s}} / k_{\mathrm{f}}=1.0, R e_{1}=0.01$, and $\varepsilon=0.5$. The results are for $10^{2} \leqslant P e_{1} \leqslant 5 \times 10^{3}$

\begin{tabular}{lccccc}
$\langle v\rangle_{\mathrm{v}} /\langle u\rangle_{\mathrm{V}}$ & $a_{2}$ & $a_{1}$ & $\langle v\rangle_{\mathrm{v}} /\langle u\rangle_{\mathrm{v}}$ & $a_{2}$ & $a_{1}$ \\
\hline 0 & 1.71 & 0.048 & 0.9 & 1.17 & 0.045 \\
0.01 & 1.25 & 0.44 & 1 & 1.17 & 0.049 \\
0.05 & 0.97 & 0.807 & 2 & 1.15 & 0.031 \\
0.1 & 0.97 & 0.403 & 4 & 1.13 & 0.024 \\
0.3 & 1.15 & 0.050 & 6 & 1.12 & 0.020 \\
0.5 & 1.30 & 0.010 & 10 & 1.10 & 0.018 \\
0.7 & 1.25 & 0.020 & 100 & 0.98 & 0.074 \\
\hline
\end{tabular}


In order to simulate random porous media, we use the ensemble averaging of the flow direction (i.e. tilt angle) for the in-line arrangement of cylinders. We use a uniform probability distribution function for the flow direction distribution and take the ensemble average of $D_{\|} / \alpha_{f}$, for the same Peclet number, using the results for the different tilt angles shown in Table 2 . Using these averaged values, we find that the computed coefficients $a_{1}$ and $a_{2}$ are 0.062 and 1.17 , respectively. This $a_{2}$ is close to that found for $\langle v\rangle_{\mathrm{v}} /\langle u\rangle_{\mathrm{V}}=1$, i.e. the average tilt angle, and it is also close to the experimental results for random arrangement of spheres (i.e. $1 \leqslant a_{2} \leqslant 1.2$ ).

\subsection{Transverse component}

The variables that most noticeably affect $D_{\perp}^{\mathrm{d}} / \alpha_{\mathrm{f}}$ are : the Peclet number, the particle arrangement, and the flow direction. The numerical results show that for a given $P e_{1}, D_{1}^{d} / \alpha_{\mathrm{f}}$ is independent of whether $\operatorname{Pr}$ or $R e_{i}$ is varied. Also, the porosity and the particle shape (i.e. square vs circular cylinders) do not affect $D_{\perp}^{\mathrm{d}} / \alpha_{\mathrm{f}}$ (but the effective conductivity depends in these parameters for $k_{\mathrm{s}} / k_{\mathrm{f}} \neq 1$ ).

4.2.1. Particle arrangement and Peclet number. In the staggered arrangement, the fluid particles follow a more tortuous path and this enhances $D_{\perp}^{\mathrm{d}} / \alpha_{\mathrm{f}}$. This is evident in Fig. 5(a), where for the staggered arrangement $D_{\perp} / \alpha_{\mathrm{f}}$ increases more rapidly with the Peclet number (as compared to in-line). For the nearly rectilinear flow fields of the in-line arrangement, the heat transfer in the transverse direction occurs only by diffusion. The only dispersion mechanism of heat transfer in the transverse direction is the recirculation which provides some mixing of the flow. For the inline arrangement and at high $P e_{1}\left(P e_{1}=10^{3}\right)$ the increase in $D_{1} / \alpha_{\text {f }}$ due to this mixing is about $35 \%$. Note that $D_{L}^{\mathrm{d}} / \alpha_{\mathrm{f}}$ for the staggered arrangement is still small compared to $D_{\perp}^{\mathrm{d}} / \alpha_{\mathrm{f}}$, because there is no net flow in the $y$-direction.

4.2.2. Flow direction. The effect of flow direction (with respect to the principal axes) on $D_{\perp}^{\mathrm{d}} / \alpha_{f}$, for inline arrangement of circular cylinders, is shown in Fig. 5(b). For a given $P e_{1_{x}}$ and when the velocity in the transverse direction is small compared to that in the longitudinal direction (e.g. $\langle v\rangle_{V} /\langle u\rangle_{V}=0.1$ ), $D_{\perp}^{\mathrm{d}} / \alpha_{\mathrm{f}}$ is slightly larger than that for the zero tilt angle. As the velocity in the transverse direction increases, $D_{i}^{\mathrm{d}} / \alpha_{\mathrm{f}}$ is increased further. For $\langle v\rangle_{V} /\langle u\rangle_{V}=1$ a substantial increase in the transverse hydrodynamic dispersion is found as expected, since the in-line arrangement is a staggered arrangement in the oblique direction of the flow.

\section{NEAR BOUNDING SURFACES}

Near the bounding surfaces of porous media, the distribution of the solid phase is significantly different than that in the bulk and this will influence $D_{\downarrow}$, because both $k_{\mathrm{e} \perp}$ and $D_{\perp}^{\mathrm{d}}$ depend on the solid phase distribution. As with the hydrodynamics and the con- (o)
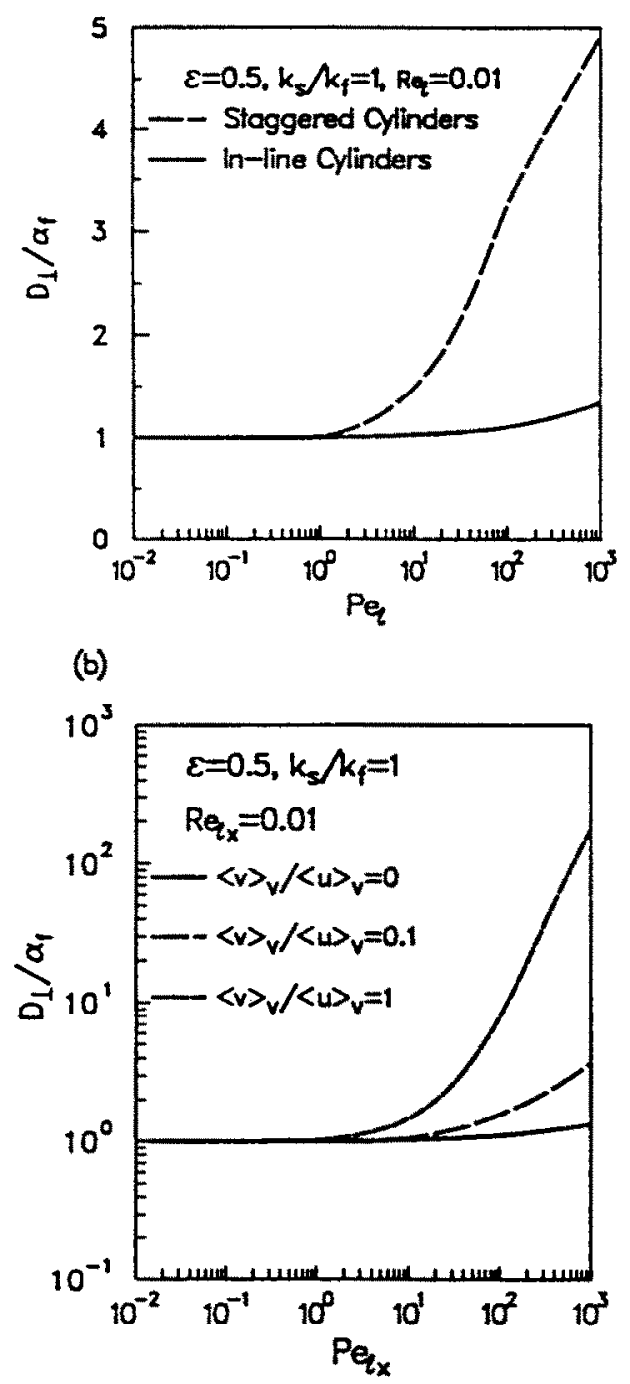

FiG. 5. (a) Effect of the particle arrangement on $D_{1} \alpha_{f}$ for inline and staggered arrangements of cylinders. (b) Effect of the flow tilt angle on $D_{1} / \alpha_{\mathrm{f}}$ for the in-line arrangement of circular cylinders.

duction heat transfer treatments (refs. $[20,21]$ ), this nonuniformity in $D_{\perp}$ can be either allowed (i.e. no temperature slip is allowed) or masked by using an average and uniform $D_{1}$ (i.e. a slip in temperature is allowed). Here we examine the effect of the bounding medium (i.e. solid or fluid) on the nonuniformity of $D_{\perp} / \alpha_{\mathrm{f}}$ using the in-line arrangement of circular cylinders. We also examine the effects of $P e_{l}, h$, and $k_{\mathrm{s}} / k_{\mathrm{r}}$ on the distribution and magnitude of $D_{1}(y) / \alpha_{f}$.

\subsection{Temperature slip boundary condition}

Since generally the variation of $D_{\perp}$ near the interface is not known, the empirical slip boundary condition is used. This boundary condition uses the extrapolation of the temperature fields away from the 
interface and an empirical slip coeffcient $x_{T}$. The slip boundary condition, based on the temperature gradient in the porous medium, is

$$
\left.\frac{\mathrm{d}\langle T\rangle_{\mathrm{V}}}{\mathrm{d} y}\right|_{y=0}=\alpha_{\mathrm{T}}\left(T^{-\cdots}-T^{+}\right)
$$

where the temperatures $T^{+}$and $T$ at the interface are found by the extrapolation of the temperature fields away from the interface (i.e. where the boundary-layer effects are not present). The slip coefficient is calculated using

$$
\alpha_{\mathrm{T}}=\frac{\left.\frac{\mathrm{d}\langle T\rangle_{\mathrm{v}}}{\mathrm{d} y}\right|_{1=0^{-}}}{\left(T^{-}-T^{+}\right)} .
$$

The computed area- and volume-averaged local temperatures are shown in Fig. 6(a) for $P_{e_{1}}=10^{2}$, $k_{\mathrm{s}} / k_{\mathrm{f}}=1$, and $\varepsilon=0.48$. The thermal boundary layer in the fluid bounding medium contributes to the temperature jump more significantly, compared to the boundary layer in the porous medium. The thermal boundary layer in the plain medium is due to the local two dimensionality of the flow. As $P e_{1}$ increases, this boundary layer effect becomes more significant. Due to the mixing in the recirculation region between the cylinders, the flow in the bed side also contributes, but slightly, to the slip in temperature. The results for $T^{--}-T^{+}$and $\alpha_{\mathrm{T}}$ are given in Table 3, for different Peclet numbers and for a given gap size $h$. The results show that as the Peclet number increases, the slip in the temperature becomes noticeable and can be larger

(a)

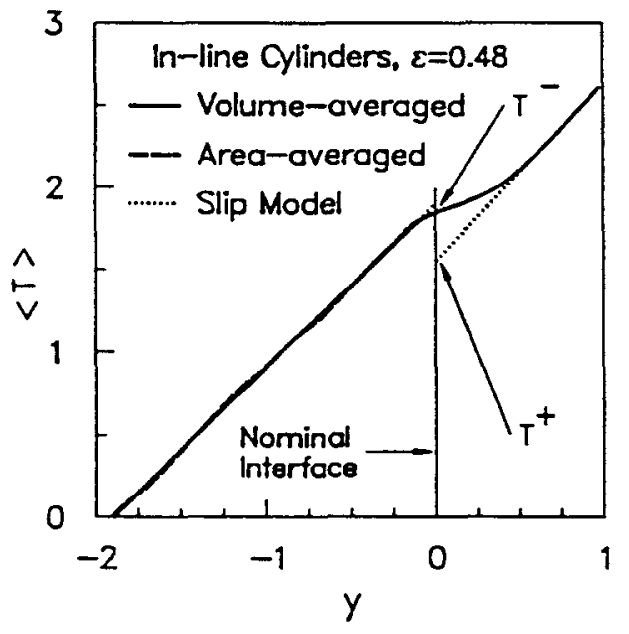

(b)

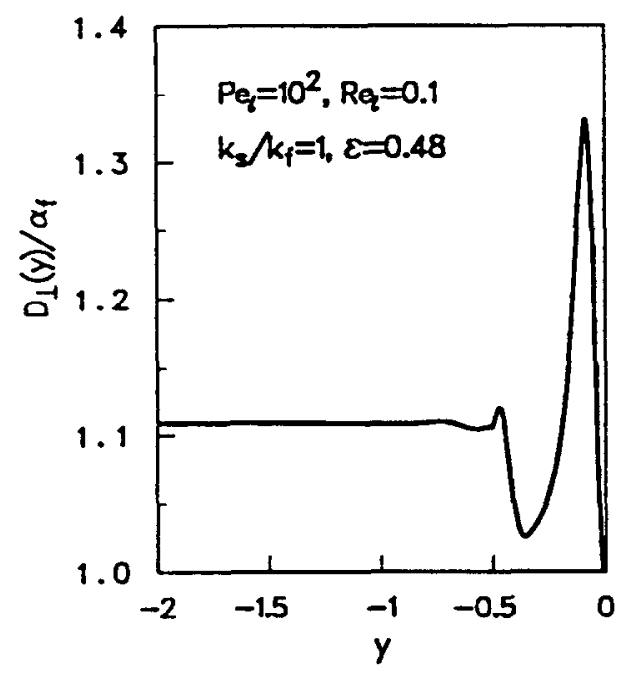

(c)

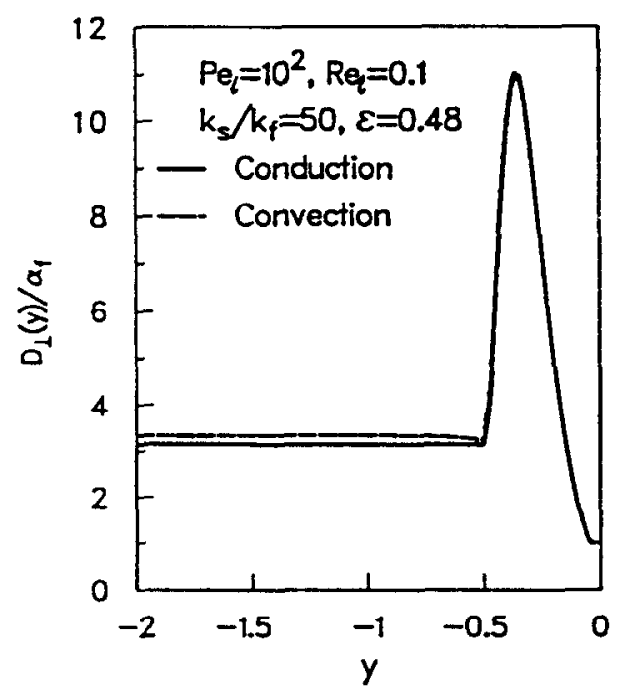

FIG. 6. (a) Distribution of the volume- and area-averaged temperature and the distribution of the extrapolated temperatures for the slip model $\left(\varepsilon=0.48, R e_{1}=0.1, P e_{1}=10^{2}\right.$, and $\left.k_{\mathrm{s}} / k_{\mathrm{f}}=1\right)$. (b) Distribution of $D_{\perp}(y) / \alpha_{f}$ near the solid bounding surface. (c) Effect of $k_{\mathrm{s}} k_{\mathrm{f}}$ on $D_{\perp}(y) / \alpha_{\mathrm{f}}$ near the solid bounding surface. 
Table 3. Effect of the Peclet number on the slip coefficient $\left(k_{\mathrm{s}} / k_{\mathrm{f}}=1.0, R e_{1}=0.1, \varepsilon=0.48\right.$, and $h=2$ )

\begin{tabular}{ccc}
$P e_{i}$ & $T^{-}-T^{+}$ & \multicolumn{1}{c}{$\alpha_{\mathrm{T}}$} \\
\hline 10 & 0.10 & 10.62 \\
$10^{2}$ & 0.72 & 1.55 \\
$10^{3}$ & 1.55 & 0.89 \\
\hline
\end{tabular}

than the temperature difference across one cell (in the bulk of the porous medium). Thus, if a uniform $D_{\perp} / \alpha_{f}$ is used along with the no-slip condition, a significant error results in the computed interfacial heat flux. From the results on conduction heat transfer given in ref. [21], we expect this error to become larger as $k_{\mathrm{s}} / k_{\mathrm{f}}$ becomes significantly different than unity. Note that for small Peclet numbers, i.e. $P_{e_{1}}<10$, conduction dominates the lateral heat transfer and the results for the slip conditions reported in ref. [21] apply.

The effect of $h$ on $\alpha_{T}$ is similar to that of $P e_{1}$. This is because as $h$ increases, the velocity near the interface also increases. A more detailed discussion of the effect of the gap size on the local flow near the interface is given in ref. [20], where the effect of $h$ on the hydrodynamic slip boundary condition is examined. The effect of $h$ on the total temperature slip is demonstrated in Table 4 for $\varepsilon=0.48$ and $P e_{1}=10^{2}$. For $h>3, T-T^{+}$reaches an asymptotic value near unity. This is because the penetration of the boundary effect in the plain medium reaches an asymptote for $h>3$. The slip coefficient decreases with increasing $h$, because the temperature slip increases while the gradient of the volume-averaged temperature remains the same.

\subsection{No-slip boundary condition}

In the no-slip temperature boundary condition, a variable $D_{\perp}$ is used in order to model the nonuniformity near the interface. In this local simulation, $D_{\perp}(y)$ is computed using the volume-averaged transverse heat flow and the gradient of the volume-averaged temperature, i.e.

$$
-\frac{D_{\perp}(y)}{\alpha_{\mathrm{f}}} \frac{\mathrm{d}\langle T\rangle_{\mathrm{v}}}{\mathrm{d} y}=\left\langle P e_{1} v T-\frac{k}{k_{\mathrm{f}}} \frac{\partial T}{\partial y}\right\rangle_{\mathrm{v}}
$$

In the $x$-direction, periodic boundary conditions are used and no heat flow occurs in that direction. The transverse heat flow is the same as the volume-averaged heat flow, i.e.

Table 4. Effect of the gap size on the slip coefficient $\left(k_{\mathrm{s}} / k_{\mathrm{f}}=1.0, R e_{1}=0.1, \varepsilon=0.48\right.$, and $\left.P e_{1}=10^{2}\right)$

\begin{tabular}{rcc}
$h$ & $T^{-} T^{+}$ & $\alpha_{\mathrm{T}}$ \\
\hline 1 & 0.35 & 3.09 \\
2 & 0.72 & 1.55 \\
3 & 0.77 & 1.45 \\
4 & 0.82 & 1.37 \\
\hline
\end{tabular}

$$
\left\langle P e_{l} v T-\frac{k}{k_{\mathrm{f}}} \frac{\partial T}{\partial y}\right\rangle_{\mathrm{v}}=\left\langle-\frac{\partial T}{\partial y}\right\rangle_{\mathrm{A}_{x}}(y=h) .
$$

Note that at the upper boundary we have $v(x, h)=0$. Then, from equations (29) and (30), $D_{\perp}(y) / \alpha_{\mathrm{f}}$ is found using

$$
\frac{D_{\perp}(y)}{\alpha_{\mathrm{f}}}=\frac{\left\langle\frac{\partial T}{\partial y}\right\rangle_{\mathrm{A}_{x}}(y=h)}{\frac{\mathrm{d}\langle T\rangle_{v}}{\mathrm{~d} y}(y)} .
$$

The variation of $D_{\perp}(y) / \alpha_{\mathrm{f}}$ obtained using equation (31), is discussed below for solid and fluid bounding media.

5.2.1. Dispersion near a solid bounding surface. We first examine $D_{\perp}(y) / \alpha_{\mathrm{f}}$ for a solid bounding surface and for $k_{\mathrm{s}} / k_{\mathrm{f}}=1$. Since the gap size is part of the porous medium, $h$ is chosen such that the last cylinder row also has a porority of 0.48 (i.e. corresponding to the simple cubic arrangement for spheres). Here the interface is taken as the bounding surface (instead of the surface tangent to the tip of the cylinders). The results for $D_{\perp}(y) / \alpha_{f}$ presented in Fig. 6(b), for $P e_{1}=10^{2}$ and $k_{s} / k_{f}=1$, show that the boundary effect only penetrates half of a cell size into the porous medium (a similar trend is found in ref. [21] for conduction heat transfer). Near the bounding surface, $D_{\perp}(y) / \alpha_{f}$ undergoes a peak and further away from the bounding surface, and before reaching the bulk value, $D_{\perp}(y) / \alpha_{\mathrm{f}}$ decrcases to values lower than the bulk value. The decrease in $D_{\perp}(y) / \alpha_{\mathrm{f}}$ is due to the recirculation region between the cylinders. In the previous studies reviewed in ref. [5], $D_{\perp}^{\mathrm{d}}(y) / \alpha_{\mathrm{f}}$ is correlated using the local volume-averaged velocity $\langle u\rangle_{\mathrm{V}}$ and a mixing length and these cause the maximum in $D_{\perp}^{\mathrm{d}}(y) / \alpha_{\mathrm{f}}$ to be located further away from the surface compared to the location of the maximum of the local velocity. We use the computed $\langle u\rangle_{\vee}(y)$ and $D_{\perp}(y)$ to calculate the mixing length and we find a distribution which is different than the linear distribution suggested by Cheng and Vortmeyer [1] or the exponential decaying function suggested by Cheng and Hsu [2]. The distribution for the mixing length is given in ref. [24].

The effect of $k_{\mathrm{s}} / k_{\mathrm{f}}$ on $D_{\perp}(y) / \alpha_{\mathrm{f}}$ is shown in Fig. 6(c) for $k_{\mathrm{s}} / k_{\mathrm{r}}=50$ and $P e_{1}=10^{2}$. For small $|y|$ where the averaging volume does not enclose any solid, $D_{1}(y) / \alpha_{f}$ is unity. As $|y|$ increases, the contribution of the transverse effective conductivity $k_{\mathrm{e} \perp}(y) / k_{\mathrm{f}}$ becomes more significant compared to the hydrodynamic effect. The results presented in Fig. 6(c) for convection and conduction are decomposed and the only significant difference between the two exists for $y<-0.4$. This significant dominance of conduction is also evident in the experiments of Yagi and Kunii [8]. Their results show that the temperature slip, for their random packed bed of spheres, is nearly the same for the stagnant and flowing air. Therefore, accurate modeling of $k_{\mathrm{e} \perp}(y) / k_{\mathrm{f}}$ is more important in predicting 
the heat transfer across the bounding surface. In the previous studies mentioned above and reviewed in ref. [5], $k_{\mathrm{e} \perp}(y) / k_{\mathrm{r}}$ is found using the local porosity and an effective conductivity-porosity correlation (for packed beds of spherical particles). Using the model for $k_{\mathrm{e}:}(y) k_{\mathrm{r}}$ used by Cheng and $\mathrm{Hsu}$, in the onedimensional energy equation, and comparing the results with the experimental results of Yagi and Kunii, we find that this local effective conductivity predicts a lower heat flux at the interface. Therefore, in the previous studies such as Cheng and $\mathrm{Hsu}$, the empirical constants introduced in modeling $D_{\perp}^{\mathrm{d}}(y) / \alpha_{\text {, }}$ are used mostly to correct the deficiency in predicting $k_{\mathrm{e} \perp}(y) / k_{\mathrm{f}}$.

5.2.2. Dispersion near a bounding channel flow: For a porous medium bounded by a fluid bounding medium, the nonuniformity in $D_{\perp}(y) / \alpha_{\mathrm{f}}$ is influenced by the flow in the channel. This is shown in Fig. 7(a), where for $k_{\mathrm{s}} / k_{\mathrm{f}}=1$ the effect of the thermal boundary layer in the plain medium (which depends on $P P_{1}$ ) is rather dominant. Away from the interface, in the porous medium this Peclet number dependence is very weak. As the Peclet number vanishes, $D_{!} / \alpha_{\mathrm{f}}$ becomes uniform for $k_{\mathrm{s}} / k_{\mathrm{f}}=1$. For $k_{\mathrm{s}} / k_{\mathrm{f}} \neq 1$, the nonuniformity in $D_{\perp}(y) / \alpha_{i}$ is present on both sides of the interface, as shown in Fig. 7(b). The nonuniformity in $D_{l}(y) / \alpha_{\mathrm{f}}$ in the porous medium depends mostly on the magnitude of $k_{\mathrm{s}} / k_{\mathrm{f}}$ and again modeling of $k_{\mathrm{e} \perp} / k_{\mathrm{f}}$ becomes more important than $D_{\perp}^{\mathrm{d}} / \alpha_{\mathrm{f}}$.

The effect of $h$ on the variation of $D_{\perp} / \alpha_{f}$ is shown in Fig. $7(\mathrm{c})$, where an increase in $h$ increases the local Peclet number near the interface and then given an increase in $D_{1} / \alpha_{f}$. For $h \geqslant 3$, as shown in Fig. 7(c), the nonuniformity in $D_{1} / x_{1}$ extends to about one eell size in the plain medium. The penetration depth in the plain medium is independent of $h$, however, the magnitude of $D_{\perp}(y) / \alpha_{f}$ increases with $h$.

\section{SUMMARY}

In order to determine the effect of the bed par-

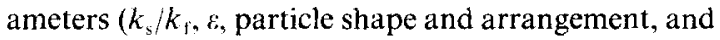
$P r$ ) and flow parameters (the direction of the Darcean flow with respect to the principal axes of the solid matrix and $R e_{1}$ ) on the dispersion tensor, both near the bounding surfaces and in the bulk, a numerical two-dimensional direct simulation is performed. The summary of the numerical results is given below.

\subsection{Dispersion far from the interface}

- A near $P e_{1}^{2}$ dependency of $D_{\|} / \alpha_{f}$ only occurs for the in-line arrangement of particles, where the flow field is similar to that for a variable diameter tube (even though recirculation regions are present between the particles) and when the fluid particles follow a nontortuous path. When the particles are arranged orderly but in a staggered manner, the fluid particle path becomes tortuous and this dependency approaches $P e_{1}$. The absence of any recirculation in the staggered arrangement also contributes to this difference in behavior. This near $P e_{1}$ dependency of $D / x_{f}$ has also been observed experimentally by others for thombohedral arrangement of spheres.

- Every other parameter being the same, the magnitude of $D / \alpha_{1}$ is smaller for the staggered arrangement, while $D_{\perp} / \alpha_{i}$ is larger.

- For the in-line arrangement, an increase in the flow tilt angle increases $D / \alpha_{\mathrm{f}}$ and decreases $D_{\|} / \alpha_{\mathrm{f}}$. As the tilt angle changes the exponent of the $P e_{1}$ dependency changes between 0.97 and 1.7. The $P e_{1}$ dependency for random porous media is obtained using an ensemble average over all tilt angles and a $\boldsymbol{P}_{\boldsymbol{e}_{1}^{1.17}}$ dependency is obtained. This is similar to that found in the existing experiments with random arrangement of spheres.

- The effect of $k_{\mathrm{s}} / k_{\mathrm{f}}$ on $\mathbf{D}$ varies with $P e_{\text {, }}$, at low $P e_{1}$ an increase in $k_{\mathrm{s}} k_{\mathrm{f}}$ results in an increase in $D_{\mid}$, while for high $P e_{i}$ this is reversed.

- Lumping the effect of $P r$ and $R e_{1}$ in $P e_{1}$ masks the hydrodynamic effects of $\mathbf{D}$ and this can be significant.

\subsection{Dispersion near the interface}

- Thermal boundary layers grow on both sides of the interface, with the plain medium boundary layer being more significant for $k_{s} / k_{\mathrm{f}}=1$. For $k_{\mathrm{s}} / k_{\mathrm{f}}>1$, the boundary layer effects in the porous medium also become significant.

- The total temperature slip increases with $h, P_{l}$, and $k_{\mathrm{s}} / k_{\mathrm{f}}$.

- Variation of $D_{\perp} / \alpha_{f}$ shows a peak in the plain medium near the interface and a strong dependence on $h$ and $P e_{1}$.

- For a solid bounding surface, $D_{\perp}(y) / \alpha_{\mathrm{f}}$ shows a strong dependency on $k_{\mathrm{s}} / k_{\mathrm{f}}$. The hydrodynamic effects are not significant, and therefore, the proper description of the variation of $k_{\mathrm{c} \perp} / k_{\mathrm{f}}$ becomes crucial.

- For $k_{\mathrm{s}} / k_{\mathrm{f}}=1$, modeling of $D_{\perp}^{\mathrm{d}}(y) / \alpha_{\mathrm{f}}$ by using the local velocity and a simple monotonic distribution of the mixing length does not lead to accurate predictions. For $k_{\mathrm{s}} / k_{\mathrm{f}}>1$, the hydrodynamic contribution is not significant and $D_{\mathrm{L}}^{\mathrm{d} / \alpha_{\mathrm{f}}}$ can be neglected compared to $k_{\mathrm{e} L} / k_{\mathrm{f}}$.

The porous, plain media interfacial conditions for velocity [20] and temperature (conduction [21] and convection here) show that the boundary layers extend only one half of a cell size in the porous medium. For a fluid bounding the porous medium, the velocity boundary in the plain medium is negligibly small but for the thermal boundary effect is very important and this increases with increase in the Peclet number $P e_{1}$ and gap size $h$. The thermodynamic slip coefficient $\alpha$ is greatly affected by $\varepsilon, R e_{1}, h$, the prescribed interfacial position (other than nominal), surface structure, and the Darcean flow direction and the results show that $0.1<\alpha<4$. For the hydrodynamic no-slip boundary condition, the distributions of the variable effective viscosity and the variable permeability cannot be readily generalized. For conduction, the slip coefficient $\alpha_{\mathrm{T}}$ depends on $\varepsilon, k_{\mathrm{s}} / k_{\mathrm{f}}$, and 


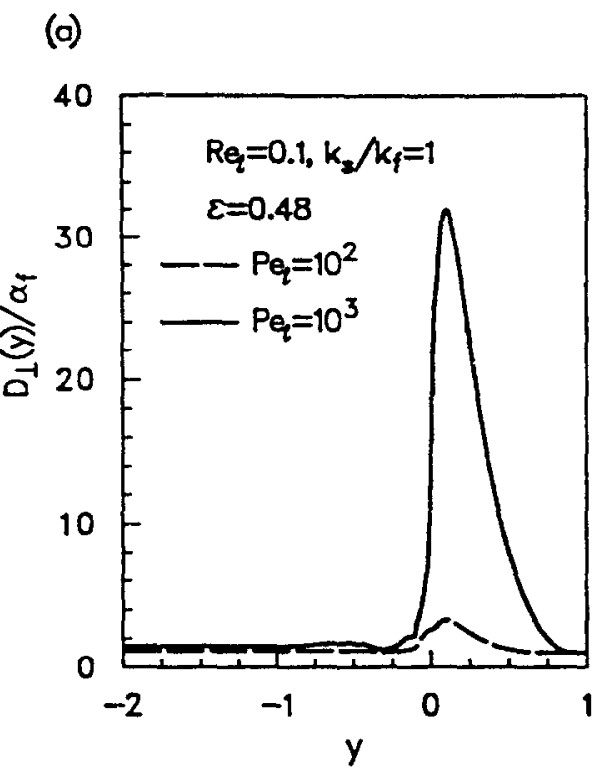

(b)

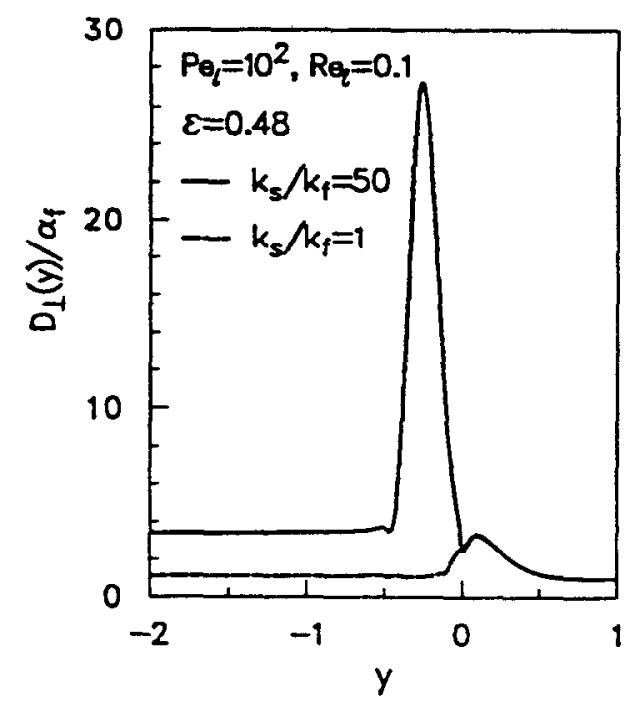

(c)

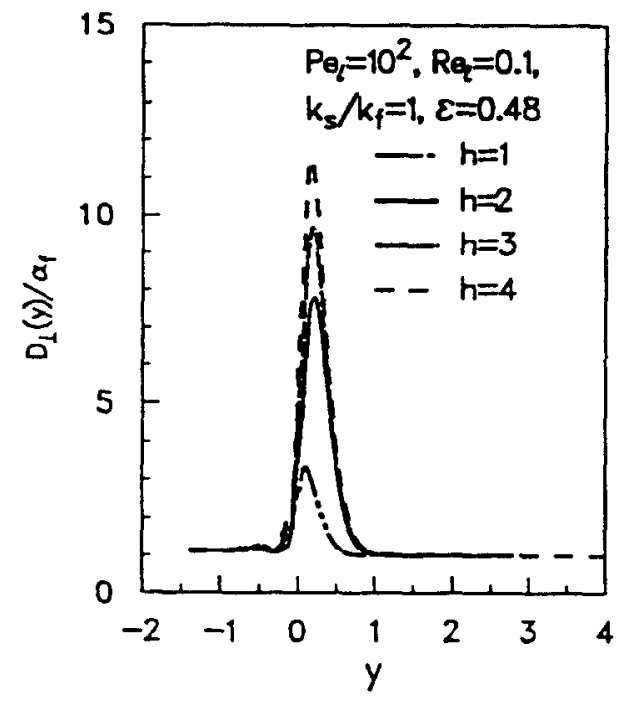

Fig. 7. (a) Distribution of $D_{\perp}(y) / \alpha_{\uparrow}$ near the interface of a bed of cylinders with the fluid bounding medium for $P e_{1}=10^{2}$ and $P e_{1}=10^{3}(h=1)$. (b) Effect of $k_{\mathrm{s}} / k_{\mathrm{f}}$ on the distribution of $D_{\perp}(y) / \alpha_{\mathrm{f}}$ for the fluid bounding medium with $k_{\mathrm{s}} / k_{\mathrm{f}}=1$ and $k_{\mathrm{s}} / k_{\mathrm{f}}=50(h=2)$. (c) Effect of the gap size on the distribution of $D_{\perp}(y) / \alpha_{\mathrm{f}}$ for $h=1,2,3,4$.

the normalized conductivity of the solid bounding medium $k_{\mathrm{s}_{\mathrm{b}}} / k_{\mathrm{f}}$ and for the no-slip boundary condition the distributions of $k_{\mathrm{e} \perp}(y) / k_{\mathrm{f}}$ and $k_{\mathrm{e} \|}(y) / k_{\mathrm{f}}$ cannot be readily generalized. The distribution of $k_{\mathrm{e} \perp}(y) / k_{\mathrm{f}}$ is most accurately modeled by the existing layered model. For convection, the results are summarized above and the importance of the proper modeling of $k_{\mathrm{e} \perp}(y) / k_{\mathrm{f}}$ near the interface should be emphasized. We point out that an error in the transverse heat flux results if the local velocity distribution and a simple mixing length distribution are used to model the distribution of $D_{\perp}^{\mathrm{d}}(y) / \alpha_{f}$.

\section{REFERENCES}

1. P. Cheng and D. Vortmeyer, Transverse thermal dispersion and wall channelling in a packed bed with forced convective flow, Chem. Engng Sci.43, 2523-2532 (1988).

2. P. Cheng and C. T. Hsu, Fully-developed forced convective flow through an annular packed-sphere bed with 
wall effects, Int. J. Heat Mass Transier 29, $1843-1853$ (1986).

3. J. Tobis and D. Ziolkowski, Modeling of heat transfer at the wall of a packed-bed apparatus, Chem. Engng Sci. 43, 3031-3036 (1988).

4. C. T. Hsu and P. Cheng, Thermal dispersion in a porous medium, Int. J. Heat Mass Transfer 33, 1587-1597 (1990)

5. M. Sahraoui and M. Kaviany, Slip and no-slip temperature boundary conditions at interface of porous, plain media: convection, Part 1. formulation, 1992 National Heat Transfer Conference, San Diego, California HTD-Vol. 193, pp. 25-33 (1993).

6. M. Kaviany, Principles of Heat Transfer in Porous Media. Springer, New York (1991).

7. G. S. Beavers and D. D. Joseph, Boundary conditions at a naturally permeable wall, $J$. Fluid Mech. 30, 197 . 207 (1967).

8. S. Yagi and D. Kunii, Studies on heat transfer near wall surface in packed beds, A.I.Ch.E. J/6, 97-104 (1960).

9. K. Ofuchi and D. Kunii, Heat transfer characteristics of packed beds with stagnant fluids, Int. J. Heat Mass Transfer 8, 749-757 (1965).

10. G. I. Taylor, Dispersion of soluble matter in solvent flowing slowly through a tube, Proc. R. Soc. L.ond. A219, 186-203 (1953).

1I. G. I. Taylor, The dispersion of matter in turbulent flow through a pipc, Proc. R. Soc. Lond. A223, 446-477 (1954).

12. D. L. Koch, R. G. Cox, H. Brenner and J. F. Brady, The effect of order on dispersion in porous media, J. Fluid Mech. 200, 173-188 (1989).

13. H. Brenner, Dispersion resulting from flow through spatially periodic porous media, Phil. Trans. R. Soc. Lond. 297, 81-133 (1980)

14. D. L. Koch and J. F. Brady, Dispersion in fixed beds, $J$. Fluid Mech. 154, 399-427 (1985).
15. R. G. Carbonell and S. Whitaker, Dispersion in pulsed systems-II. Theoretical developments for passive dispersion in porous media, Chem. Engng Sci. 38, 1795 1802 ( I983).

16. R. G. Carboncll and S. Whitaker, I Ieat and mass transfer in porous media. In Fundamentals of Transport Phenomena in Porous Media (Edited by J. Bear and M. Y. Corapcioglu), pp. $121 \ldots$ [98. Martinus Nijhoff, Dordrecht (1984).

17. A. Eidsath, R. G. Carbonell, S. Whitaker and L. R. Herman, Dispersion in pulsed systems--III. Comparison between theory and experiments for packed beds, Chem. Engng Sci. 38, 1803-1816 (1983).

18. D. J. Gunn and C. Pryce, Dispersion in packed beds, Trans. Inst. Chem. Enynrs 47, T341 T350 (1969).

19. D. A. Edwards, M. Shapiro, H. Brenner and M. Shapira, Dispersion of inert solutes in spatially periodic, twodimensional model porous media, Transp. Porous Media 6, $338 \cdots 358$ (1991).

20. M. Sahraoui and M. Kaviany, Slip and no-slip velocity boundary conditions at interface of porous, plain media, Int. J. Heat Mass Transfer 35, 927-943 (1992).

21. M. Sahraoui and M. Kaviany, Slip and no-slip temperature boundary conditions at interface of porous, plain media : conduction, Int. J. Heat Mass Jransfer 36, 10191033 (1993).

22. S. V. Patankar, Numerical Heat Transfer and Fluid Flow. Hemisphere, Washington, D.C. (1980).

23. M. Quintard, Private communication (1992)

24. M. Sahraoui and M. Kaviany, Slip and no-slip temperature boundary conditions at interface of porous, plain media : convection, Part 2. Results, 1992 National Heat Transfer Conference, San Diego, California, HTDVol. 193, pp. 3447 (1993).

25. Z. Yuan, W. Somerton and K. S. Udell, Thermal dispersion in thick wall tubes as a model of porous media, Int. J. Heat Mass Transfer 34, 2715-2726 (1991) 